\title{
Generative Type Abstraction and Type-level Computation (Extended Version)
}

\author{
Stephanie Weirich \\ University of Pennsylvania \\ Philadelphia, PA, USA \\ sweirich@cis.upenn.edu
}

\author{
Dimitrios Vytiniotis \\ Simon Peyton Jones \\ Microsoft Research \\ Cambridge, UK \\ \{dimitris,simonpj\}@microsoft.com
}

\author{
Steve Zdancewic \\ University of Pennsylvania \\ Philadelphia, PA, USA \\ stevez@cis.upenn.edu
}

\begin{abstract}
Modular languages support generative type abstraction, ensuring that an abstract type is distinct from its representation, except inside the implementation where the two are synonymous. We show that this well-established feature is in tension with the non-parametric features of newer type systems, such as indexed type families and GADTs. In this paper we solve the problem by using kinds to distinguish between parametric and non-parametric contexts. The result is directly applicable to Haskell, which is rapidly developing support for type-level computation, but the same issues should arise whenever generativity and non-parametric features are combined.
\end{abstract}

Categories and Subject Descriptors $\quad$ D.3.3 [Language Constructs and Features]: Abstract data types; F.3.3 [Studies of Program Constructs]: Type structure

General Terms Design,Languages

Keywords Haskell, Newtype deriving, Type functions

\section{Introduction}

Generative type abstraction allows programmers to introduce new type constants in their programs that are isomorphic to existing types; examples include ML's module system [Milner et al. 1997; Pierce 2005, Ch. 8], and Haskell's newtype construct [Peyton Jones et al. 2003]. Type generativity is important because it supports modularity by enforcing abstraction: the implementor of a module can move freely between the abstract and representation types, whereas to the client of the module the two types are completely distinct.

There is growing interest in languages that support some form of type-level computation including Haskell's type classes [Hall et al. 1996] and indexed type families [Kiselyov et al. 2010]. However, there is a fundamental tension between type-level computation and generative type abstraction, at least in the latter's more flexible forms. To summarize very briefly, the conflict is this:

- To maximize re-use and convenience, it is very desirable for the implementor to be able to treat the abstract type $A$ and its

[Copyright notice will appear here once 'preprint' option is removed.] concrete representation type $C$ as synonymous - we call this flexible type generativity.

- However, given type-level function $F$ the result of $(F A)$ and $(F C)$ may differ, so $A$ and $C$ cannot be synonymous.

Resolving this conflict is the subject of this paper. Specifically our contributions are:

- We show in Section 2 that the naive combination of type generativity and non-parametric type-level features can violate soundness; a problem that already manifests in the Glasgow Haskell Compiler, and affects not only type-level non-parametric functions, but also other forms of non-parametric constructs, such as generalized algebraic datatypes (GADTs) [Cheney and Hinze 2003; Hinze et al. 2002; Peyton Jones et al. 2006; Xi et al. 2003].

- We formalize a solution to this problem that reconciles flexible type generativity and non-parametric type functions in Section 3. Our language, $\mathrm{FC}_{2}$, builds on GHC's existing core language, System FC [Sulzmann et al. 2007], which supports erasable type-level coercions. The key ingredient in our solution is to employ kinds decorated with roles to mark the distinctions that type contexts may make.

- We prove that $\mathrm{FC}_{2}$ programs are type safe, provided that user axioms and definitions give rise to consistent axiom sets (Section 4).

- We give sufficient conditions for showing the consistency of an axiom set. For our proofs, we introduce a rewrite system for types that is novel in two dimensions: rewriting (i) is role-sensitive, and (ii) need not be strongly normalizing (Section 4.2).

- We present a Haskell-specific result: we show how Haskell source programs that may involve non-parametric type contexts and flexible type generativity can be translated to yield provably consistent $\mathrm{FC}_{2}$ axiom sets. (Section 5)

- Our core language is an improvement of System FC since it permits safe flexible type generativity, but also unsaturated type functions. Perhaps surprisingly, our language is additionally a significant simplification of the original System FC, which removes several of the original coercion constructs that we have identified to be encodable (even in the original System FC). We discuss these differences in Section 6.2.

For the sake of concreteness we build our presentation around Haskell and System FC, since this setting allows us to demonstrate our points with real code, instead of using a hypothetical $\lambda$-calculus. However, we stress that our work is applicable when- 
ever flexible type generativity and non-parametric type-level features are combined. For example, the very same issues could arise in extensions of the ML module system. We discuss related work in Section 7.

\section{The problems with generative type abstraction}

Generative type abstraction is an extremely useful mechanism for enforcing abstraction barriers and for refining interfaces. To see this benefit, let us consider the Haskell incarnation of type generativity, namely newtype definitions. In Haskell, the programmer may declare a newtype Age, with concrete representation type Int, thus:

$$
\text { newtype Age }=\text { MkAge Int }
$$

The implementor uses the "data constructor" MkAge to coerce an Int to an Age, and pattern matching to effect the inverse coercion. For example:

$$
\begin{aligned}
& \text { addAge }:: \text { Age } \rightarrow \text { Int } \rightarrow \text { Age } \\
& \text { addAge (MkAge a) } \mathrm{n}=\text { MkAge }(a+n)
\end{aligned}
$$

The client, in contrast, can be prevented from making such conversions, by using the module system to hide the MkAge constructor:

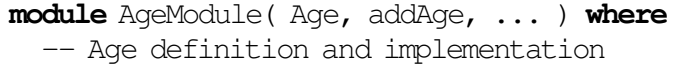

\subsection{Coercion lifting}

In describing MkAge, we wrote "data constructor" in quotes because although it behaves in many ways like a data constructor, its cost model is different. Specifically, a newt ype definition guarantees that the abstract type really is represented by the concrete type, so the runtime conversion cost is zero. That would not be true if Age were instead declared with data instead of newtype.

So Age can be coerced to an Int, and vice versa, for free-i.e. without runtime cost-because it is an Int. Notationally, we say that Int $\sim$ Age, where we use $\sim$ for type equality ${ }^{1}$. But what about, say, Maybe Age?

$$
\text { data Maybe } \mathrm{a}=\text { Nothing } \mid \text { Just a }
$$

Obviously, Maybe Age should be freely coercible to Maybe Int, because the two are represented identically. Alas, in Haskell 98 one would have to write

$$
\begin{aligned}
& \text { cvtAge }:: \text { Maybe Age } \rightarrow \text { Maybe Int } \\
& \text { cvtAge } t=\text { mapMaybe }(\lambda(\text { MkAge a) } \rightarrow \text { a) } t \\
& -- \text { mapMaybe }::(a \rightarrow b) \rightarrow \text { Maybe a } \rightarrow \text { Maybe b }
\end{aligned}
$$

This is unsavory for several reasons: (a) it is tedious for the programmer; (b) it may be difficult to implement the necessary "map" function; (c) it is hard for the compiler to eliminate a runtime call to the map function (let alone to guarantee to do so) especially if the map function is recursive. As an example of (b) consider the mapping function for the type $\mathrm{T}$ shown below, with co- and contravariance, and higher kinds:

$$
\begin{aligned}
& \text { data } \mathrm{T} \text { a } \mathrm{f}=\mathrm{T} 1 \text { a } \\
& \qquad \begin{array}{l}
\mathrm{T} 2(\mathrm{a} \rightarrow \mathrm{Int}) \\
\mathrm{T} 3 \quad(\mathrm{f}(\mathrm{T} \text { a } \mathrm{f}) \quad(\mathrm{T} \text { a } \mathrm{f}))
\end{array}
\end{aligned}
$$

These difficulties are frustrating, because we know that, say, $\mathrm{T}$ Age Maybe is represented identically to $\mathrm{T}$ Int Maybe. So, let us imagine a hypothetical extension of Haskell that provides lifted coercions; that is, it implements the following rule:

\footnotetext{
${ }^{1}$ There are too many sorts of " $="$ !
}

Coercion lifting: if for two types $\varphi$ and $\psi$ we have $\varphi \sim \psi$ (for example, if they are the abstract and concrete types of a newtype declaration), then $T \varphi \sim T \psi$ for any type constructor $T$.

In fact this extension is not so hypothetical, because such a rule is the basis of the so-called "newtype deriving" feature implemented by GHC. It is not difficult to use newtype deriving to define an identity function whose type is

cvt $:: \forall\{c\}$. c Int $\rightarrow c$ Age

ML supports coercion lifting even more directly: within a structure the abstract and the representation types are considered entirely interchangeable. For example in Standard ML one might say

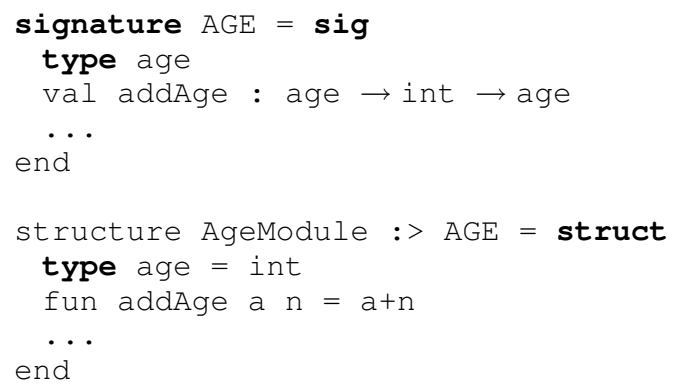

Inside AgeMo du le the two types are synonymous, and so addAge need not convert in either direction.

However, the point of this paper is that the innocuous and obvious-seeming "lifting" of type identities becomes unsound when combined with type-level computation, as we show in the next section.

\subsection{Type-level computation}

One very popular extension to Haskell is that of Generalized Algebraic Data Types (GADTs) [Peyton Jones et al. 2006], with which we assume the reader is somewhat familiar. In GHC one could declare a GADT with two nullary constructors like this:

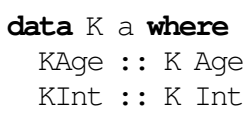

Now, consider these definitions (using cvt from Section 2.1):

$$
\begin{array}{ll}
\text { kint }:: \text { K Age } & \text { get }:: \mathrm{K} \text { Age } \rightarrow \text { String } \\
\text { kint }=\text { cvt KInt } & \text { get KAge }=\text { "Age" }
\end{array}
$$

Since get's type signature declares that its argument is of type $\mathrm{K}$ Age, the patterns in get are exhaustive. But consider the call (get kint). It is patently well typed, yet the pattern match in get will fail (recall that cvt is operationally the identity function), and if the compiler assumed otherwise a runtime crash could result.

In the last few years we have gone beyond GADTs, by extending GHC with type-level functions [Chakravarty et al. 2005a,b; Kiselyov et al. 2010]. The reader is urged to consult these papers for motivated examples of type functions, but for the purposes of this paper we content ourselves with a small but contrived example:

type family $\mathrm{F}$ a : : *

type instance $\mathrm{F}$ Age $=$ Char

type instance $\mathrm{F}$ Int $=\mathrm{BoOl}$

Here the type function $\mathrm{F}$ maps the type Age to the type Char, but it maps Int to Bool.

However, the existence of such a type-level function threatens not just pattern exhaustiveness but type soundness itself. Consider the type Bool. This type is equivalent to $\mathrm{F}$ Int by the equation 
for $\mathrm{F}$; and by coercion lifting that should be equivalent to $\mathrm{F}$ Age; and that is equivalent to Char by the other equation for $\mathrm{F}$. Altogether we can coerce Bool to Char, which is obvious nonsense.

What went wrong? Maybe it should be illegal for a type function to behave differently on two coercible types, such as Age and Int? But in fact Haskell programmers often use newtypes precisely so that they can give a different type-class instance (for comparison, say) for Age than for the underlying Int. Type functions are no different; indeed, they are often introduced as an "associated type" of a type class [Kiselyov et al. 2010], and hence, just as the type class distinguishes between the abstract and concrete type, so must the type function.

How else might we fix the problem? Perhaps, in the definition of coercion lifting we should not allow $T$ to range over type functions such as F or GADTs such as K? Indeed we should not, but that is not enough. Consider

$$
\text { data } \mathrm{TF} \text { a }=\operatorname{MkTF}(\mathrm{F} \text { a) }
$$

Now, should coercion lifting allow us to coerce TF Age to $\mathrm{TF}$ Int? Obviously not! Otherwise we could write

$$
\begin{array}{ll}
\text { to }:: \mathrm{Bool} \rightarrow \mathrm{TF} \text { Int } & \text { from }: \mathrm{TF} \text { Age } \rightarrow \text { Char } \\
\text { to } \mathrm{b}=\mathrm{MkTF} \mathrm{b} & \text { from }(\mathrm{MkTF} \mathrm{c})=\mathrm{c}
\end{array}
$$

and now the composition from o cvt o to is well-typed (via coercion lifting) but obviously unsound. So in the the definition of coercion lifting, as well as type functions and GADTs we must exclude data types like TF that use their type arguments nonparametrically.

\subsection{Summary}

At this point it should be clear that a naive combination of:

- type-level dispatch, whether by GADTs or by type functions

- unrestricted coercion lifting

simply does not work. This interaction was far from obvious to us initially, and indeed GHC has a well-documented type-soundness bug $^{2}$ that arises directly from this unforeseen interaction. Yet both type-level dispatch and coercion lifting (suitably restricted) are valuable. The purpose of this paper is to show how they may be soundly combined.

This problem is important not only because it arises in GHC (which is our main source of motivation), but also because the same issues will arise in any type system that combines type-level dispatch and coercion lifting. Haskell is the first programming language that has pushed the type system far enough for these problems to arise in practice, but others may do so in the future. However, these different languages may expose the coercions between abstract and concrete types in different ways, possibly explicitly (as in Haskell) or implicitly (as in ML), in a way that is intimately connected with type inference. To avoid the complications associated with type inference, in this paper, we focus on an intermediate language, in which (runtime-erasable) coercions are explicit. Whether they come directly from the source program, or from elaboration by the type checker, is secondary.

\section{The $\mathrm{FC}_{2}$ language: codes versus types}

Our goal is to resolve the tension between generative types, which allow programmers to express the intent that two types have identical representations, and type functions, which can distinguish two types even if they have the same underlying representation.

The inspiration for our solution comes from encodings of generic programming in dependently typed languages [Benke et al.

\footnotetext{
${ }^{2}$ http://hackage.haskell.org/trac/ghc/ticket/1496
}

2003; Dybjer 2000]. These encodings use "codes" to represent types as a form of data. For example, the code Int Code may be the code for the type Int. Nonparametric functions branch on codes and thus have a different type from parametric functions. In this context, we can view Age and Int Code as two different codes that both map to the type Int. However, this encoding requires significant syntactic overhead as we must have a code for every type and must explicitly map codes to types when they are used to classify expressions.

However, the important distinction between codes and types is that they have different definitions of equality. In the encoding above, the codes Age and Int Code are different codes, but their interpretations are equal types. Therefore, we use this idea in $\mathrm{FC}_{2}$ to define roles, which support different notions of equality for the same data. In this language, Age and Int are distinct when viewed at role code but equal when viewed at role type. Code equality is used to reason about the meaning of type-indexed functions and is finer-grained than type equality, which is used to determine which type coercions are safe. Importantly, $\mathrm{FC}_{2}$ distinguishes type functions by what equivalences they respect. Parametric functions respect the role type, whereas functions that distinguish between Age and Int do not.

\section{1 $\mathrm{FC}_{2}$ : an overview}

These ideas are best explained in terms of an intermediate language that exposes the differences between the code and type roles and makes explicit the uses of the two kinds of equality mentioned above. Thus, the remainder of this section describes $\mathrm{FC}_{2}$, our new variant of System FC [Sulzmann et al. 2007] - a model of the intermediate language used in GHC. As such, it is expressive enough to capture indexed type functions, newtype and newtype deriving, GADTs, existential and nested datatypes, and much more.

Figure 1 summarizes the syntax of $\mathrm{FC}_{2}$, which, at the term level $(e)$, is just the polymorphic lambda calculus with two extensions. First, $\mathrm{FC}_{2}$ provides polymorphic datatypes, introduced by data constructors $K$. These datatypes are eliminated using a case construct that should be familiar from Haskell or ML-style functional programming-we describe how datatypes and case are typechecked in Section 3.5.

Second, $\mathrm{FC}_{2}$ includes first-class proofs of type equality that witness safe coercions introduced during compilation. Programs in $\mathrm{FC}_{2}$ can abstract over coercions reflecting a particular type equality (written $\Lambda c: \varphi_{1} \sim \varphi_{2} . e$ ), pass a coercion as an argument to such a function (written $e \gamma$ ), and use a coercion to cast a term from one type to another (written $e \triangleright \gamma$ ). These explicit coercions, written $\gamma$, make typechecking $\mathrm{FC}_{2}$ programs syntax-directed-that is, the syntax of an $\mathrm{FC}_{2}$ term encodes its typing derivation. Why is this important? The idea is that the compiler's front end performs perhaps-complex type inference on the source program, and records the proofs generated by inference directly in the syntax of the $\mathrm{FC}_{2}$ intermediate language. The optimiser transforms $\mathrm{FC}_{2}$ terms, perhaps radically. At any point one can check the consistency of the resulting $\mathrm{FC}_{2}$ program using a simple, fast, syntaxdirected typechecker; this consistency check has proven to be an extremely powerful aid to getting the compiler right. It is just as easy to find the type of an arbitrary $\mathrm{FC}_{2}$ term, an ability that is used extensively inside GHC.

Figure 2 shows the typing rules for the terms of $\mathrm{FC}_{2}$. The first five rules, EEVAR through ETAPP, are completely standard. We defer explanation of the remaining rules until we build up technical machinery having to do with $\mathrm{FC}_{2}$ 's kind-level distinction between codes and types and the rules by which explicit coercions can themselves be combined. We describe these aspects of $\mathrm{FC}_{2}$ next. 


\begin{tabular}{|c|c|c|c|}
\hline$\eta$ & $::=$ & $\star \mid \kappa \rightarrow \eta$ & kind \\
\hline$R$ & $::=$ & $\mathrm{C} \mid \mathrm{T}$ & role \\
\hline$\kappa$ & $::=$ & $\eta / R$ & kind and role \\
\hline$H$ & $::=$ & $\begin{array}{l}T \\
F \\
(\rightarrow) \\
\left(\sim_{\eta}\right)\end{array}$ & $\begin{array}{l}\text { type constants } \\
\text { datatypes } \\
\text { functions/newtypes } \\
\text { arrow } \\
\text { equality }\end{array}$ \\
\hline$\varphi, \sigma, \psi$ & $::=$ & $\begin{array}{l}a \\
H \\
\varphi_{1} \varphi_{2} \\
\forall a: \kappa . \varphi\end{array}$ & $\begin{array}{l}\text { codes and types } \\
\text { variables } \\
\text { constants } \\
\text { application } \\
\text { polymorphism }\end{array}$ \\
\hline$\gamma$ & $::=$ & $\begin{array}{l}c \bar{\varphi} \\
\varphi \\
\text { sym } \gamma \\
\gamma_{1} ; \gamma_{2} \\
\gamma_{1} \gamma_{2} \\
\text { nth } k \gamma \\
\forall a: \kappa \cdot \gamma_{2} \\
\gamma @ \varphi\end{array}$ & $\begin{array}{l}\text { coercion proof } \\
\text { assumption } \\
\text { reflexivity } \\
\text { symmetry } \\
\text { transitivity } \\
\text { application } \\
\text { injectivity } \\
\text { polymorphism } \\
\text { instantiation }\end{array}$ \\
\hline$e$ & $::=$ & $\begin{array}{l}x \\
\lambda x: \sigma . e \\
e_{1} e_{2} \\
\Lambda a: \kappa . e \\
e \varphi \\
K \\
\operatorname{case}_{\sigma} e \text { of } b r s \\
\Lambda c: \varphi_{1} \sim \varphi_{2} . e \\
e \gamma \\
e \triangleright \gamma\end{array}$ & $\begin{array}{l}\text { expressions } \\
\text { variable } \\
\text { abstraction } \\
\text { application } \\
\text { type abstraction } \\
\text { type application } \\
\text { data constructor } \\
\text { case analysis } \\
\text { proof abstraction } \\
\text { proof application } \\
\text { coercion }\end{array}$ \\
\hline brs & $::=$ & ${\overline{K_{i} \Rightarrow e_{i}}}^{i \in 1 . . n}$ & branches \\
\hline bnd & $::=$ & $\begin{array}{l}a: \kappa \\
H: \eta \\
c: \Delta . \varphi_{1} \sim \varphi_{2} / R \\
x: \sigma \\
K: \sigma\end{array}$ & $\begin{array}{l}\text { binding } \\
\text { type variable } \\
\text { type constant } \\
\text { coercion } \\
\text { term variable } \\
\text { data constructor }\end{array}$ \\
\hline$\Gamma$ & $::=$ & $\cdot \mid \Gamma, b n d$ & context \\
\hline$\Delta$ & $::=$ & $\cdot \mid a: \kappa, \Delta$ & type context \\
\hline$\rho$ & $::=$ & $e|\varphi| \gamma$ & datacon argument \\
\hline$\Theta$ & $::=$ & $\begin{array}{l}\sigma, \Theta \\
a: \kappa, \Theta \\
\varphi_{1} \sim \varphi_{2}, \Theta\end{array}$ & $\begin{array}{l}\text { telescopes } \\
\text { empty } \\
\text { expression type } \\
\text { type variable } \\
\text { equality }\end{array}$ \\
\hline$v$ & $:=$ & $\begin{array}{l}\lambda x: \sigma . e \mid \Lambda a: \kappa . e \\
\Lambda c: \varphi_{1} \sim \varphi_{2} . e \\
K \bar{\varphi} \bar{\rho}\end{array}$ & values \\
\hline$\tau$ & $::=$ & $\begin{array}{l}T|(\rightarrow)|\left(\sim_{\eta}\right) \\
\forall a: \kappa \cdot \varphi \mid \tau \varphi\end{array}$ & value types \\
\hline
\end{tabular}

Figure 1. Syntax
$\Gamma \vdash e: \sigma$

$$
\begin{aligned}
& \frac{x: \sigma \in \Gamma \quad \vdash \Gamma}{\Gamma \vdash x: \sigma} \quad \text { EEVAR } \\
& \frac{\Gamma, x: \sigma_{1} \vdash e: \sigma_{2}}{\Gamma \vdash \lambda x: \sigma_{1} \cdot e: \sigma_{1} \rightarrow \sigma_{2}} \quad \text { EEABS } \\
& \frac{\Gamma \vdash e_{1}: \sigma_{1} \rightarrow \sigma_{2} \quad \Gamma \vdash e_{2}: \sigma_{1}}{\Gamma \vdash e_{1} e_{2}: \sigma_{2}} \quad \text { EEAPP } \\
& \frac{\Gamma, a: \kappa \vdash e: \sigma}{\Gamma \vdash \Lambda a: \kappa . e: \forall a: \kappa \cdot \sigma} \quad \text { ETABS } \\
& \frac{\Gamma \vdash e: \forall a: \kappa . \sigma \quad \Gamma \vdash \varphi: \kappa}{\Gamma \vdash e \varphi: \sigma[a \mapsto \varphi]} \quad \text { ETAPP } \\
& T: \bar{\kappa} \rightarrow \star \in \Gamma \\
& \frac{K: \forall \bar{a}: \bar{\kappa} \cdot \forall \Theta . T \bar{a} \in \Gamma \quad \vdash \Gamma}{\Gamma \vdash K: \forall \bar{a}: \bar{\kappa} \cdot \forall \Theta . T \bar{a}} \quad \text { EDATACON } \\
& \Gamma \vdash e: T \bar{\varphi} \quad \Gamma \vdash \sigma: \star / \top \\
& \text { for each } K_{i} \in \operatorname{Constr}_{\Gamma}(T) \\
& K_{i}: \forall \bar{a}: \bar{\kappa} \cdot \psi_{i} \in \Gamma \\
& \psi_{i}[\bar{a} \mapsto \bar{\varphi}]=\forall \Theta_{i} . T \bar{\varphi} \\
& \Gamma \vdash e_{i}: \forall \Theta_{i} . \sigma \\
& {\overline{\Gamma \vdash \text { case }_{\sigma} \text { e of }} \overline{K_{i} \Rightarrow e_{i}}}^{i}: \sigma \\
& \text { ECASE } \\
& \frac{\Gamma, c: \varphi_{1} \sim \varphi_{2} / \mathrm{C} \vdash e: \sigma}{\Gamma \vdash \Lambda c: \varphi_{1} \sim \varphi_{2} . e:\left(\varphi_{1} \sim \varphi_{2}\right) \Rightarrow \sigma} \quad \text { ECABS } \\
& \Gamma \vdash e:\left(\varphi_{1} \sim \varphi_{2}\right) \Rightarrow \sigma \\
& \frac{\Gamma \vdash \gamma: \varphi_{1} \sim \varphi_{2} \in \eta / \mathrm{C}}{\Gamma \vdash e \gamma: \sigma} \quad \text { ECAPP } \\
& \frac{\Gamma \vdash e: \sigma_{1} \quad \Gamma \vdash \gamma: \sigma_{1} \sim \sigma_{2} \in \star / \mathrm{T}}{\Gamma \vdash e \triangleright \gamma: \sigma_{2}} \quad \text { ECOERCE }
\end{aligned}
$$

Figure 2. Typing rules

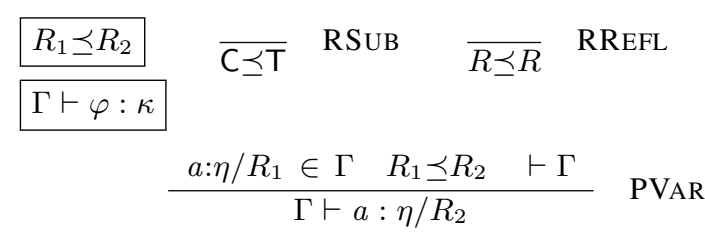$$
\frac{H: \eta \in \Gamma \quad \vdash \Gamma}{\Gamma \vdash H: \eta / R} \quad \text { PConst }
$$$$
\Gamma \vdash \varphi_{1}:\left(\eta_{1} / R_{2} \rightarrow \eta_{2}\right) / R_{1}
$$$$
\frac{\Gamma \vdash \varphi_{2}: \eta_{1} / \min \left(R_{1}, R_{2}\right)}{\Gamma \vdash \varphi_{1} \varphi_{2}: \eta_{2} / R_{1}} \quad \text { PAPP }
$$$$
\frac{\Gamma, a: \kappa \vdash \varphi: \star / R}{\Gamma \vdash \forall a: \kappa \cdot \varphi: \star / R} \quad \text { PALL }
$$

$\Gamma \vdash \bar{\varphi}: \bar{\kappa}$

$$
\overline{\Gamma \vdash \cdot:} \quad \text { PNIL } \quad \frac{\Gamma \vdash \varphi: \kappa \quad \Gamma \vdash \bar{\varphi}: \bar{\kappa}}{\Gamma \vdash \varphi, \bar{\varphi}: \kappa, \bar{\kappa}} \quad \text { PCONS }
$$

Figure 3. Kinding 


\section{2 $\quad \mathrm{FC}_{2}$ types and kinds}

Types in $\mathrm{FC}_{2}$ are classified by pairs $\kappa$ of the form $\eta / R$, where the kind $\eta$ ensures (as usual) that types are well-formed structurally, and the role $R$ that determines the precision at which they can be analyzed. Codes (which distinguish Age and Int) have role C, whereas types (which identify them) have role $T$. This syntax is summarized at the top of Figure 1, while the kinding rules are in Figure 3.

The distinction between codes and types allows us to give informative kinds to type constructors:

- The Maybe type (Section 2.1) has kind $\star / T \rightarrow \star$, indicating that Maybe treats its argument parametrically.

- The types K, F, and TF (Section 2.2) all use type indexing and therefore have kind $\star / C \rightarrow \star$.

These kinds in turn support the key insight of this paper: it is only safe to lift coercions through functions with parametric kinds. So Maybe Age $\sim$ Maybe Int holds but TF Age $\not$ TF Int.

The rules for constructing coercions are given in Section 3.3, but first we must deal with the kinding rules for types. The critical rule is PAPP in Figure 3, which deals with type application. It is quite conventional except for the treatment of roles. Consider these type declarations:

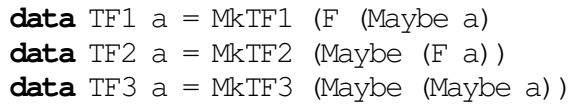

In the first two cases, type variable a is used non-parametrically (intuitively, under a type function), so TF1 : $\star / C \rightarrow \star$, and similarly for $\mathrm{TF} 2$. In contrast, $\mathrm{TF} 3$ treats a parametrically, so TF $1: \star / T \rightarrow \star$. The unusual "min" in rule PAPP achieves these kindings by combining the role of the context and the argument role of the function to get the role of the argument.

In fact, $\min$ is just the least upper bound induced by an inclusion relation $C \preceq T$ on roles (see the top of Figure 3 ). This inclusion makes explicit the fact that code equality implies type equality but not vice-versa. For example, consider

data $\mathrm{TF} 4$ a $=\operatorname{MkTF} 4$ (F a) a

Here $a$ is used both non-parametrically (as an argument of $F$ ) and parametrically (as a plain argument of $\mathrm{MkTF} 4$ ). So a has role $\star / C$, which makes sense; just because a type can be analyzed does not mean that it must be.

Despite these non-standard kinds, the types of $\mathrm{FC}_{2}$ are mostly standard. Codes $\varphi$ and types $\sigma$ are drawn from the same syntax (see Figure 1), although we use two different metavariables as a reminder of the intended role. The type language includes type variables $a$, type constants $H$, applications $\varphi_{1} \varphi_{2}$, and polymorphic types $\forall a: \kappa . \sigma$.

Type constants, $H$, include datatypes $T$, and type functions $F$. For the most part, datatypes and type functions are treated uniformly, but there are two important distinctions:

- Datatypes must be injective, while type functions need not be. Injectivity is important because equalities between applications of injective functions imply equalities between their arguments; see rule CNTHT in Section 3.3.

- Datatypes are inhabited by values, but type functions are notthere are no values $v$ with types that are headed by $F$. (There are coerced values with such types, but no raw values.) This distinction is key to the definition of consistency in Section 4.1.

Newtypes are not inhabited by raw values, so we treat them like type functions, ranged over by $F$. Unlike type functions, however, newtypes are injective at role $\mathrm{C}$ - after all, the essence of generativity is that newtypes create a fresh constant-but for now we will not take advantage of that fact, leaving it for discussion in Section 6.1.

The set of type constants also includes the familiar arrow type constructor $(\rightarrow)$, and a kind-indexed family of constructors $\left(\sim_{\eta}\right)$, which construct functions that abstract over coercions. The kinds of these constants are:

$$
\begin{array}{ll}
(\rightarrow) & : \quad \star / \mathrm{T} \rightarrow \star / \mathrm{T} \rightarrow \star \\
\left(\sim_{\eta}\right) & : \quad \eta / \mathrm{C} \rightarrow \eta / \mathrm{C} \rightarrow \star / \mathrm{T} \rightarrow \star
\end{array}
$$

(We discuss in Sections 3.3 and 3.6 why the kind of $\left(\sim_{\eta}\right)$ must require role $\mathrm{C}$ for its first two arguments.) Type constants are generally applied prefix, but for these two constants we define infix syntactic sugar:

$$
\begin{aligned}
& \sigma \rightarrow \sigma^{\prime} \quad \equiv(\rightarrow) \sigma \sigma^{\prime} \\
& \left(\varphi_{1} \sim \varphi_{2}\right) \Rightarrow \sigma \equiv\left(\sim_{\eta}\right) \varphi_{1} \varphi_{2} \sigma
\end{aligned}
$$

In the latter case, because the syntactic sugar lacks the $\eta$ annotation, we only use this notation in contexts where the kind of $\varphi_{1}$ and $\varphi_{2}$ is irrelevant. Rule ECABS follows this convention-it shows that this family of type constructors is used to give a type to terms of the form $\Lambda c: \varphi_{1} \sim \varphi_{2} . e$, which abstracts over an equality proof in the body $e$. Note that this rule applies only to code equalities; abstraction over type equalities is not needed for compilation of Haskell and permitting it, while straightforward, would require extra syntactic complexity.

Figure 3 defines the kinding rules for $\mathrm{FC}_{2}$ using judgments of the form $\Gamma \vdash \varphi: \kappa$. Here, the context $\Gamma$ maps type variables to their kind/role pairs and type constants and type functions to their kinds. This judgment relies on a judgment $\vdash \Gamma$ (shown in the appendix) that checks that contexts are well formed. These checks ensure that constants $(\rightarrow)$ and $\left(\sim_{\eta}\right)$ have the right kinds, that data constructors and term variables have well-formed types and that coercion axioms introduce equalities between well-formed codes and types.

Rule PCONST allows a type constant to play either role. Although a type constant may be given role $\mathrm{C}$, its kind may well involve arguments with role $\mathrm{T}$; for example, see the signature for $(\rightarrow)$ above. It follows that closed types can play both roles, independently of whether they contain newtypes or type functions. For example (Int, Age) : $\star / C$ and (Int, Age) $: \star / T$ both hold.

Together these kinding rules implement a subsumption relation that includes codes into the language of types:

LEMMA 1. If $\Gamma \vdash \varphi: \eta / \mathrm{C}$ then $\Gamma \vdash \varphi: \eta / \mathrm{T}$.

On the other hand, types have only one kind regardless of their role:

LEMMA 2. If $\Gamma \vdash \varphi: \eta_{1} / R_{1}$ and $\Gamma \vdash \varphi: \eta_{2} / R_{2}$ then $\eta_{1}=\eta_{2}$.

Note that the kinding judgment, like term typing, is syntaxdirected (see the remarks in Section 3.1). In particular, the role component $R$ of the $\kappa$ in this judgment is treated as input to the algorithm, and the $\eta$ is an output - the context in which $\varphi$ is used determines what the role should be. The only interesting case from this perspective is PAPP, in which $\varphi_{1}$ must be checked first to obtain $R_{2}$ so that the minimum of $R_{1}$ and $R_{2}$ can be passed as an input when checking $\varphi_{2}$.

\subsection{Coercions and equality}

In $\mathrm{FC}_{2}$ a coercion is a proof term that witnesses the equality of two types. Coercions are used to change the type of a term, thus (Figure 2):

$$
\frac{\Gamma \vdash e: \sigma_{1} \quad \Gamma \vdash \gamma: \sigma_{1} \sim \sigma_{2} \in \star / T}{\Gamma \vdash e \triangleright \gamma: \sigma_{2}} \quad \text { ECOERCE }
$$

Here, $\gamma$ is a coercion witnessing the equality $\sigma_{1} \sim \sigma_{2}$ in role $\mathrm{T}$; given that $e$ has type $\sigma_{1}$, we can use $\gamma$ to let us treat the term as hav- 


$$
\begin{aligned}
& \Gamma \vdash \gamma: \varphi_{1} \sim \varphi_{2} \in \kappa \\
& \left(c: \bar{a}: \bar{\kappa} \cdot \varphi_{1} \sim \varphi_{2} / R_{1}\right) \in \Gamma \\
& \frac{\Gamma \vdash \varphi_{1}: \eta / R_{1} \quad \Gamma \vdash \bar{\psi}: \bar{\kappa} \quad R_{1} \preceq R_{2}}{\Gamma \vdash c \bar{\psi}: \varphi_{1}[\bar{a} \mapsto \bar{\psi}] \sim \varphi_{2}[\bar{a} \mapsto \bar{\psi}] \in \eta / R_{2}} \quad \text { CAssM } \\
& \frac{\Gamma \vdash \varphi: \kappa}{\Gamma \vdash \varphi: \varphi \sim \varphi \in \kappa} \quad \text { CREFL } \\
& \frac{\Gamma \vdash \gamma: \varphi_{1} \sim \varphi_{2} \in \kappa}{\Gamma \vdash \operatorname{sym} \gamma: \varphi_{2} \sim \varphi_{1} \in \kappa} \quad \operatorname{CSYM} \\
& \Gamma \vdash \gamma_{1}: \varphi_{1} \sim \varphi_{2} \in \kappa \\
& \frac{\Gamma \vdash \gamma_{2}: \varphi_{2} \sim \varphi_{3} \in \kappa}{\Gamma \vdash \gamma_{1} ; \gamma_{2}: \varphi_{1} \sim \varphi_{3} \in \kappa} \quad \text { CTRANS } \\
& \Gamma \vdash \gamma_{1}: \varphi_{1} \sim \varphi_{2} \in\left(\eta_{1} / R_{2} \rightarrow \eta_{2}\right) / R_{1} \\
& \frac{\Gamma \vdash \gamma_{2}: \psi_{1} \sim \psi_{2} \in \eta_{1} / \min \left(R_{1}, R_{2}\right)}{\Gamma \vdash \gamma_{1} \gamma_{2}: \varphi_{1} \psi_{1} \sim \varphi_{2} \psi_{2} \in \eta_{2} / R_{1}} \quad \text { CAPP } \\
& \Gamma \vdash \gamma: T \bar{\varphi} \sim T \bar{\psi} \in \eta / \mathrm{T} \\
& T: \bar{\kappa} \rightarrow \star \in \Gamma \\
& \eta^{\prime} / R_{1}=n t h k \bar{\kappa} \quad R_{1} \preceq R_{2} \\
& \overline{\Gamma \vdash \operatorname{nth} k \gamma: \text { nth } k \bar{\varphi} \sim n t h k \bar{\psi} \in \eta^{\prime} / R_{2}} \\
& \Gamma, a: \kappa \vdash \gamma_{2}: \varphi_{1} \sim \varphi_{2} \in \star / R \\
& \overline{\Gamma \vdash \forall a: \kappa \cdot \gamma_{2}: \forall a: \kappa \cdot \varphi_{1} \sim \forall a: \kappa \cdot \varphi_{2} \in \star / R} \\
& \Gamma \vdash \gamma: \forall a: \kappa . \varphi_{1} \sim \forall a: \kappa . \varphi_{2} \in \star / R \\
& \Gamma \vdash \psi: \kappa \\
& \overline{\Gamma \vdash \gamma @ \psi:\left(\varphi_{1}[a \mapsto \psi]\right) \sim\left(\varphi_{2}[a \mapsto \psi]\right) \in \star / R} \quad \text { CINST } \\
& \Gamma \vdash \bar{\gamma}: \bar{\varphi}_{1} \sim \bar{\varphi}_{2} \in \bar{\kappa} \\
& \frac{\vdash \Gamma}{\Gamma \vdash \cdot: \sim \cdot \epsilon \cdot} \quad \mathrm{CNIL} \\
& \Gamma \vdash \gamma: \varphi_{1} \sim \varphi_{2} \in \kappa \\
& \Gamma \vdash \bar{\gamma}: \bar{\varphi}_{1} \sim \bar{\varphi}_{2} \in \bar{\kappa} \\
& \frac{\Gamma+\bar{\gamma}: \bar{\varphi}_{1} \sim \varphi_{2} \in \bar{\kappa}}{\Gamma \vdash \gamma, \bar{\gamma}: \varphi_{1}, \bar{\varphi}_{1} \sim \varphi_{2}, \bar{\varphi}_{2} \in \kappa, \bar{\kappa}} \quad \text { CCONS }
\end{aligned}
$$

Figure 4. Coercions

ing type $\sigma_{2}$. At compile time, these explicit coercions ensure that typechecking $\mathrm{FC}_{2}$ programs is completely syntax directed. Such coercions have no run-time effect: they will be erased by the compiler before the program is run. Nevertheless, $\mathrm{FC}_{2}$ 's operational semantics includes coercions, thereby allowing us to establish type safety using standard techniques (Section 3.6).

The translation of a source program into $\mathrm{FC}_{2}$ may extend the type environment $\Gamma$ with new equality axioms. For example, the Age newtype definition generates the axiom (where mkAge is a coercion constant $c$ ):

$$
\text { mkAge : Age } \sim \text { Int } / \mathrm{T}
$$

Note that ECOERCE requires $\sigma_{1}$ and $\sigma_{2}$ to be equal when considered in role $\mathrm{T}$, which is consistent with the idea that type equality determines when it is safe to coerce. On the other hand, source programs can also introduce code equalities. For example the type function $\mathrm{F}$ (Section 2.2) generates the two axioms:

$$
\begin{aligned}
& \operatorname{axF} 1: \mathrm{F} \text { Int } \sim \mathrm{Bool} / \mathrm{C} \\
& \operatorname{axF} 2: \mathrm{F} \text { Age } \sim \mathrm{Char} / \mathrm{C}
\end{aligned}
$$

However, all code axioms can be used to prove type equalities, as code equality is a refinement of type equality.
More generally we permit axiom schemes. For example, the source language declaration

type instance $\mathrm{F}$ (Maybe a) $=(\mathrm{a}, \mathrm{a})$

would create the axiom scheme

$$
\operatorname{axF} 3:(a: \star / C) . F(\text { Maybe a }) \sim(a, a) / C
$$

In general, as shown in Figure 1, the context $\Gamma$ includes bindings of the form $c: \Delta . \varphi_{1} \sim \varphi_{2} / R$ for coercion axioms. The metavariable $\Delta$ stands for a type variable context: a list of quantified type variable bindings of the form $a: \kappa$. The same binding form is used both for axioms introduced at top level, and (with empty $\Delta$ ) for local assumptions introduced in ECABS (Figure 2).

Of course it is important to know that the top-level axioms are consistent - it would be unsound to assert that Bool $\sim$ Char $/ \mathrm{T}$, for example. Section 4 gives a sufficient set of conditions for ensuring that source programs generate consistent axioms.

Next, we need a way to compose coercions together to construct other coercions. Our goal is to have rules that allow the creation of composite coercions such as:

$$
\begin{array}{ll}
\text { List mkAge } & : \text { List Age } \sim \text { List Int } / \mathrm{T} \\
\text { List axF2 } & \text { List }(\mathrm{F} \text { Int }) \sim \text { List Bool/T }
\end{array}
$$

On the other hand, the coercion formation rules should disallow the formation of a coercion of the form $\gamma_{3}: \mathrm{F}$ Age $\sim \mathrm{F}$ Int/T, which creates the unsoundness described in Section 2.2.

Figure 1 gives the syntax of coercion terms, $\gamma$, and Figure 4 gives their typing rules. Coercions are typechecked using the judgment: $\Gamma \vdash \gamma: \varphi_{1} \sim \varphi_{2} \in \eta / R$, which asserts that the type of a coercion $\gamma$ is an equality proposition $\varphi_{1} \sim \varphi_{2} \in \eta / R$. This proposition in turn implies that $\varphi_{1}$ and $\varphi_{2}$ both have kind $\eta$ and are equal in role $R$. It is technically convenient to include $\eta$ in the syntax of the judgement to enforce that both types have the same kind. However, this component is not always relevant, so we sometimes omit the $\in \eta$ part, as we have done in the examples above.

Rule CAssm instantiates an axiom scheme with types $\bar{\psi}$, using an auxiliary judgment $\Gamma \vdash \bar{\psi}: \bar{\kappa}$ defined at the bottom of Figure 3 to ensure that each variable is instantiated with a type of the matching kind and role. The notation $\bar{a}: \bar{\kappa}$ zips together a list of type variables and a list of kinds to create a type variable context $\Delta$. These two lists must have the same length for the notation to be well-defined. The notation $\varphi[\bar{a} \mapsto \bar{\psi}]$ applies a multi-substitution of the types $\bar{\psi}$ for each of the corresponding variables in the list $\bar{a}$.

Rule CREFL shows that any type $\varphi$ can be lifted to a reflexive coercion $\varphi$, while CSYM and CTRANS add symmetry and transitivity, ensuring that equality is an equivalence relation. The rules CAPP and CALL extend equality compatibly over applications and polymorphic types; their structure is analogous to the corresponding kinding rules in Figure 3. Rule CAPP is particularly important, because it implements the key coercion lifting idea we discussed in Section 2.1, using kinds to prevent the formation of the bogus coercion

$$
\text { FmkAge }: \mathrm{F} \text { Age } \sim \mathrm{F} \text { Int } / \mathrm{T}
$$

To see why, recall that $\mathrm{F}$ has kind $\star / \mathrm{C} \rightarrow \star$, but the mkAge axiom holds only at role $\mathrm{T}$ - type equalities cannot be lifted through code functions. Another example of a coercion that is correctly rejected by the application rule (because of the kind of $\left(\sim_{\eta}\right)$ ) is

$$
\left(\sim_{\star}\right) \text { mkAge Int } \sigma
$$

This coercion proves $($ Age $\sim$ Int $) \Rightarrow \sigma \sim($ Int $\sim$ Int $) \Rightarrow \sigma$, an equality that could be used to introduce a bogus assumption that Age $\sim$ Int $/ C$ and satisfy it with reflexivity for Int.

As well as composing coercions to witness the equality of bigger types, it is also essential to do the reverse: to decompose equalities over complex types to give equalities of simpler types. 
Decomposition is required by $\mathrm{FC}_{2}$ 's operational semantics ( $\mathrm{Sec}$ tion 3.6), and it also makes the language usefully more expressive. Rule CINST allows equalities between polymorphic types to be instantiated. The other, more important decomposition rule is CNTHT, which decomposes the application of a datatype constant to arguments. For example, given a coercion $\gamma$ : List Int $\sim$ List a/T we can use nth $0 \gamma$ to conclude that Int $\sim \mathrm{a} / \mathrm{T}$. The soundness of this rule depends on the fact that datatypes are injective. In general, type functions are not, and hence CNTHT is restricted to datatypes $T$.

In rule $\mathrm{CNTHT}$, the notation $T \bar{\varphi}$ abbreviates the multi-application $\left(\left(T \varphi_{1}\right) \ldots \varphi_{n}\right)$ for $\varphi_{1} \ldots \varphi_{n}$ in $\bar{\varphi}$. In the conclusion of the rule, the notation $n t h k \bar{\psi}$, accesses the $k$ th element of the sequence of types, and $n t h k \bar{\kappa}$, accesses the kind of the $k^{t h}$ variable binding. Both of these notations are undefined if $k$ is not less than the length of the sequence. The sequence of types $\bar{\kappa}$ is determined by the kind of the datatype, using the following notation.

DEFINITION 3. We define $\bar{\kappa} \rightarrow \eta$ by induction on $\bar{\kappa}$ as follows:

$$
\begin{array}{ll}
\cdot \rightarrow \eta & =\eta \\
\kappa, \bar{\kappa} \rightarrow \eta & =\kappa \rightarrow(\bar{\kappa} \rightarrow \eta)
\end{array}
$$

The coercion judgment satisfies a number of sanity checking properties. First, coercions are between well-formed types.

LEMMA 4 (Coercion regularity). If $\Gamma \vdash \gamma: \varphi_{1} \sim \varphi_{2} \in \kappa$ then $\Gamma \vdash \varphi_{1}: \kappa$ and $\Gamma \vdash \varphi_{2}: \kappa$.

Next, each coercion proof is for one pair of codes/types.

LEMMA 5 (Unique types). If $\Gamma \vdash \gamma: \varphi_{1} \sim \varphi_{2} \in \eta / R$ and $\Gamma \vdash \gamma: \varphi_{1}^{\prime} \sim \varphi_{2}^{\prime} \in \eta^{\prime} / R$ then $\varphi_{1}=\varphi_{1}^{\prime}$ and $\varphi_{2}=\varphi_{2}^{\prime}$ and $\eta=\eta^{\prime}$.

Finally, equality for Cs is a refinement of that for Ts; that is, code equality implies type equality, but not vice versa.

Lemma 6. If $\Gamma \vdash \gamma: \varphi_{1} \sim \varphi_{2} \in \eta / \mathrm{C}$ then $\Gamma \vdash \gamma: \varphi_{1} \sim \varphi_{2} \in$ $\eta / \mathrm{T}$.

\subsection{Coercion lifting}

The application rule CAPP described in the previous section allows us to lift equalities through arbitrary type constructors; that is, for all datatypes $T$ of kind $\star / T \rightarrow \star$, we have a coercion that shows $T$ Age $\sim T$ Int $\in \star / T$.

In fact, this notion of coercion lifting is not restricted to datatypes (like List), but is available for more general contexts. More precisely, given an arbitrary type $\sigma$ with free variable $a$ of kind $\star / \mathrm{T}$, we can also create a coercion $\sigma[a \mapsto$ Age $] \sim \sigma[a \mapsto$ Int $] \in \star / T$.

We create such coercions with the lifting operation. This operation replaces type variables by coercions in types to produce a new coercion, relying on the fact that the syntax of types is a subset of the syntax of coercions:

DEFINITION 7 (Lifting Operation). Define the lifting operation, written $\varphi[a \mapsto \gamma]$, by induction on $\varphi$.

$$
\begin{array}{lll}
a[a \mapsto \gamma] & =\gamma & \\
b[a \mapsto \gamma] & =b & \text { when } a \neq b \\
H[a \mapsto \gamma] & =H & \\
(\varphi \psi)[a \mapsto \gamma] & =(\varphi[a \mapsto \gamma])(\psi[a \mapsto \gamma]) & \\
(\forall b: \kappa . \sigma)[a \mapsto \gamma] & =\forall b: \kappa \cdot(\sigma[a \mapsto \gamma]) &
\end{array}
$$

The lifting operation produces a valid result as long as the role of the lifted coercion matches the role of the type variable in the type.

LEMMA 8 (Lifting). If $\Gamma, a: \eta / R \vdash \sigma: \kappa$ and $\Gamma \vdash \gamma: \varphi \sim \varphi^{\prime} \in$ $\eta / R$, then $\Gamma \vdash \sigma[a \mapsto \gamma]: \sigma[a \mapsto \varphi] \sim \sigma\left[a \mapsto \varphi^{\prime}\right] \in \kappa$.
In other words, if $a$ were used in some indexed context in $\sigma$, that is, if its role were $C$, then we would not be able to lift the coercion Age $\sim$ Int $\in \star / T$ in $\sigma$. We generalize lifting to replace multiple type variables simultaneously in the obvious way, with notation $\sigma[\bar{a} \mapsto \bar{\gamma}]$.

\subsection{Pattern matching and datatypes}

$\mathrm{FC}_{2}$ includes a formalization of recursive datatypes. These datatypes include all GHC extensions to standard datatypes: empty datatypes, nested datatypes, existential types, first-class polymorphism and GADTs. Both datatypes $T$ and data constructors $K$ must be declared in a context $\Gamma$ before they can be used. For example, using the syntax for $\Gamma$ in Figure 1, the declarations for the data constructors of List are:

$$
\begin{array}{ll}
\text { List } & \star \star / T \rightarrow \star \\
\text { Nil }: & (a: \star / \mathrm{T}) . \text { List } a \\
\text { Cons }: & (a: \star / \mathrm{T}) . a \rightarrow \text { List } a \rightarrow \text { List } a
\end{array}
$$

What about GADTs? Here's an example in Haskell:

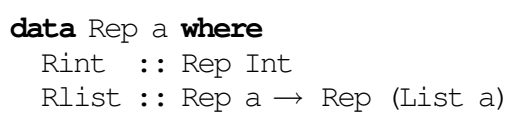

Although a Haskell programmer writes the data constructors of a GADT with non-parametric result types, in the internal type system it is simpler in the formalization for the result type of a data constructor to take the form $\left(T a_{1} \ldots a_{n}\right)$, where the $\bar{a}$ are the type parameters, using equality constraints to express the indexing, thus:

$$
\begin{aligned}
& \text { Rep }: \star \star / C \rightarrow \star \\
& \text { Rint }:(a: \star / C) \cdot(a \sim \text { Int }) \Rightarrow \operatorname{Rep} a \\
& \text { Rlist }:(a: \star / C) \cdot \forall(b: \star / C) .(a \sim \text { List } b) \Rightarrow \operatorname{Rep} b \rightarrow \operatorname{Rep} a
\end{aligned}
$$

Notice here that Rep's kind expresses that its argument is an index (role $\mathrm{C}$ ) rather than a parameter (role $\mathrm{T}$ ). In fact, the $\mathrm{C}$ role for variable $a$ falls out naturally because $a$ appears as argument to the $\left(\sim_{\star}\right)$ constructor (in the type of Rint and Rlist), which in turn requires it to have role $C$.

More generally, we use the notation of telescopes [de Bruijn 1991] to conveniently express the types of data constructors. Figure 1 defines a telescope $\Theta$ like this:

$$
\Theta::=\cdot|a: \kappa, \Theta| \varphi_{1} \sim \varphi_{2}, \Theta \mid \sigma, \Theta
$$

A telescope is like a mini-context: a list of type variable bindings, equality propositions (between codes only) and types that classify each argument of the data constructor. (Note that a type variable context $\Delta$ is also a telescope.) We define the syntactic sugar $\forall \Theta . \sigma$ as follows:

DEFINITION 9 (Telescope syntactic sugar).

$$
\begin{array}{ll}
\forall \cdot . \sigma & =\sigma \\
\forall(a: \kappa, \Theta) \cdot \sigma & =\forall a: \kappa \cdot(\forall \Theta . \sigma) \\
\forall\left(\varphi_{1} \sim \varphi_{2}, \Theta\right) \cdot \sigma & =\left(\varphi_{1} \sim \varphi_{2}\right) \Rightarrow(\forall \Theta . \sigma) \\
\forall\left(\sigma^{\prime}, \Theta\right) . \sigma & =\sigma^{\prime} \rightarrow(\forall \Theta . \sigma)
\end{array}
$$

At each use of a data constructor (rule EDATACON), we check that the declaration of a data constructor is of the form

$$
K: \forall \bar{a}: \bar{\kappa} \cdot(\forall \Theta . T \bar{a})
$$

where $\bar{a}: \bar{\kappa}$ lists the type parameters of the datatype, $\Theta$ describes the types of the arguments to the data constructor, and $T \bar{a}$ is syntactic sugar for the application of $T$ to those parameters (i.e. $\left.\left(\left(T a_{1}\right) \ldots a_{n}\right)\right)$. For example, the List type has a single parameter $a: \star / \mathrm{T}$, the constructor Cons has telescope $(a$, List $a, \cdot)$, and constructs type List $a$. Likewise, the Rep type has the parameter $a: \star / C$, the telescope for Rlist is $(b: \star / C, a \sim$ List $b,(\operatorname{Rep} b), \cdot)$, and the result type is $\operatorname{Rep} a$. 


$$
\begin{aligned}
& \gamma_{0}=\operatorname{sym}(\mathbf{n t h} 0 \gamma) \quad \gamma_{1}=\mathbf{n t h} 1 \gamma \\
& \overline{\left(\left(\lambda x: \sigma_{1} . e_{1}\right) \triangleright \gamma\right) e_{2} \rightsquigarrow\left(\lambda x: \sigma_{1} \cdot\left(e_{1} \triangleright \gamma_{1}\right)\right)\left(e_{2} \triangleright \gamma_{0}\right)} \\
& \frac{\Sigma \vdash \gamma: \forall a: \kappa . \sigma_{1} \sim \forall a: \kappa . \sigma_{2} \in \star / \mathrm{T}}{((\Lambda a: \kappa . e) \triangleright \gamma) \varphi \rightsquigarrow(\Lambda a: \kappa .(e \triangleright \gamma @ a)) \varphi} \\
& \Sigma \vdash \gamma:\left(\sim_{\eta}\right) \varphi_{1} \varphi_{2} \sigma \sim\left(\sim_{\eta}\right) \varphi_{1}^{\prime} \varphi_{2}^{\prime} \sigma^{\prime} \in \star / \mathrm{T} \\
& \gamma_{0}=\mathbf{n t h} 0 \gamma \quad \gamma_{1}=\operatorname{sym}(\text { nth } 1 \gamma) \quad \gamma_{2}=\text { nth } 2 \gamma \\
& \left(\left(\Lambda c: \varphi_{1} \sim \varphi_{2} . e\right) \triangleright \gamma\right) \gamma^{\prime} \rightsquigarrow \\
& \left(\Lambda c: \varphi_{1} \sim \varphi_{2} .\left(e \triangleright \gamma_{2}\right)\right)\left(\gamma_{0} ; \gamma^{\prime} ; \gamma_{1}\right) \\
& \Sigma \vdash \gamma: T \bar{\varphi} \sim T \bar{\varphi}^{\prime} \in \star / \mathrm{T} \\
& K: \forall \bar{a}: \bar{\kappa} . \sigma \in \Sigma \\
& \text { case }_{\sigma}^{\prime}(K \bar{\varphi} \bar{\rho}) \triangleright \gamma \text { of brs } \rightsquigarrow \\
& \operatorname{case}_{\sigma}^{\prime} K \bar{\varphi}^{\prime}(\bar{\rho} \triangleright \sigma[\bar{a} \mapsto n t h \gamma]) \text { of brs }
\end{aligned}
$$

Figure 5. Operational Semantics (Push rules)

Telescope notation is also used for case expressions in rule ECASE (Figure 2). The type of the scrutinee of the case must be headed by a datatype constant $T$. Furthermore, for each data constructor that could create a $T$, there must be a corresponding branch. The (elided) function $\operatorname{Constr}_{\Gamma}(T)$ looks up the constructors of $T$ from the context. After substituting for the parameters, the branch for data constructor $K_{i}$ must abstract the same arguments as $K_{i}$ and return the same result type as the entire case. To make sure that typechecking is syntax directed even when there are no branches (for empty datatypes), the case expression is annotated with its result type $\sigma$, and we must check that this type is well-formed in the current context.

\subsection{Operational semantics: pushing coercions}

The operational semantics of $\mathrm{FC}_{2}$ is based on a small-step callby-name operational semantics for a polymorphic lambda calculus. Although this language includes explicit type abstraction and application (as well as explicit coercion proof abstraction and application), types and coercions are not relevant at run time. All such abstractions and applications, as well as uses of coercions $e \triangleright \gamma$ may be erased prior to execution and so impose no run-time costs.

As alluded to above, this operational semantics preserves coercion proofs, which allows us to establish type safety using standard syntactic proofs of progress and preservation (described in the next section). The most important rules of the operational semantics are those that "push" coercions when they appear in active positions so that they do not interfere with reduction. Figure 5 shows the relevant pushing rules. (The complete rules of the operational semantics are listed in the appendix.) In some of the push rules, the coercion must be checked to constrain the types that it proves equal. This checking happens in a global context $\Sigma$ that contains only the definitions of data constructors, data types, and coercion axioms. Note that these checks are not actually necessary at runtime, in a consistent context these checks will always succeed.

The first three rules show how in an application of a coerced abstraction, the term steps to a new application, where the coercion has been decomposed into a coercion for the body of the abstraction, and a coercion of the argument. For example, consider SSPUSH. Here, the $\gamma$ is a coercion between some function types $\sigma_{1} \rightarrow \sigma_{1}^{\prime}$ and $\sigma_{2} \rightarrow \sigma_{2}^{\prime}$. The rule uses nth and sym to decompose $\gamma$ into two coercions, one from $\sigma_{2} \sim \sigma_{1}$ (the order is reversed to account for contra-variance) and one from $\sigma_{1} \sim \sigma_{2}^{\prime}$. These new coercions can be pushed to the body of the lambda and the function argument, exposing the reduction. Rules SSTPUSH and SSCPUSH work analogously.

$$
\begin{gathered}
\Gamma \vdash \bar{\rho}: \Theta \\
\frac{\vdash \Gamma}{\Gamma \vdash \cdot:} \quad \text { TNIL } \\
\frac{\Gamma \vdash e: \sigma \quad \Gamma \vdash \bar{\rho}: \Theta}{\Gamma \vdash e, \bar{\rho}:(\sigma, \Theta)} \quad \text { TCONSE } \\
\frac{\Gamma \vdash \varphi: \kappa \quad \Gamma \vdash \bar{\rho}: \Theta[a \mapsto \varphi]}{\Gamma \vdash \varphi, \bar{\rho}:(a: \kappa, \Theta)} \text { TCONsT } \\
\frac{\Gamma \vdash \gamma: \varphi_{1} \sim \varphi_{2} \in \eta / C \quad \Gamma \vdash \bar{\rho}: \Theta}{\Gamma \vdash \gamma, \bar{\rho}:\left(\varphi_{1} \sim \varphi_{2}, \Theta\right)} \text { TCONSC }
\end{gathered}
$$

Figure 6. Telescope rules

Note that SSCPUSH justifies the kind of $\left(\sim_{\eta}\right)$, which requires that the first two arguments be codes. If we had assigned $\left(\sim_{\eta}\right)$ the parametric kind $\eta / \mathrm{T} \rightarrow \eta / \mathrm{T} \rightarrow \star / \mathrm{T} \rightarrow \star$, then the coercions $\gamma_{0}$ and $\gamma_{1}$ in the rule would both be type coercions. However, type coercions cannot be composed with $\gamma^{\prime}$ to form a code coercion, which is the role required for the right hand side of the rule to typecheck.

The last rule, SSKPUSH, pushes the coercion of a data constructor in the scrutinee position of a case expression into coercions of the arguments of the data constructor. The arguments of a data constructor, notated $\rho$, can either be an expression, a type, or a coercion.

$$
\rho::=e|\varphi| \gamma
$$

If the declared type of the data constructor $K$ is $\forall \bar{a}: \bar{\kappa} \cdot \forall \Theta . T \bar{a}$, and the type parameters are $\bar{\varphi}$, then we know that the arguments can be typed using $\Gamma \vdash \bar{\rho}: \Theta[\bar{a} \mapsto \bar{\varphi}]$ (see Figure 6). However, pushing the coercion changes the type parameters to be $\bar{\varphi}^{\prime}$, so the new arguments must have type $\Theta\left[\bar{a} \mapsto \bar{\varphi}^{\prime}\right]$.

These new arguments are produced by coercing the list of arguments $\bar{\rho}$ with the coercion generated by lifting as described above. (The notation $\sigma[\bar{a} \mapsto n t h \gamma]$ means that variable $a_{i}$ is lifted to coercion nth $i \gamma$.) Once this coercion has been defined by lifting, we use it to coerce the list of arguments of the data constructor with the following operation.

DEFINITION 10. Define argument coercion $\bar{\rho} \triangleright \gamma$ by induction on $\bar{\rho}:$

$$
\begin{array}{ll}
\cdot \triangleright \gamma & = \\
(e, \bar{\rho}) \triangleright \gamma_{1} \rightarrow \gamma_{2} & =\left(e \triangleright \gamma_{1}\right), \bar{\rho} \triangleright \gamma_{2} \\
(\varphi, \bar{\rho}) \triangleright \forall a: \kappa \cdot \gamma_{2} & =\varphi, \bar{\rho} \triangleright \gamma_{2} \\
(\gamma, \bar{\rho}) \triangleright\left(\gamma_{1} \sim \gamma_{2}\right) \Rightarrow \gamma_{3} & =\left(\operatorname{sym} \gamma_{1} ; \gamma ; \gamma_{2}\right), \bar{\rho} \triangleright \gamma_{3}
\end{array}
$$

Argument coercion coerces a list of arguments as described by the following lemma.

LEMMA 11. If $\Gamma \vdash \bar{\rho}: \Theta$ and $\Gamma \vdash \gamma: \forall \Theta . \sigma \sim \forall \Theta^{\prime} . \sigma^{\prime} \in \star / T$ then $\Gamma \vdash(\bar{\rho} \triangleright \gamma): \Theta^{\prime}$

\section{Type safety and consistency}

The $\mathrm{FC}_{2}$ language supports a straightforward proof of type safety based on the usual preservation and progress theorems. Importantly, the progress theorem holds only for consistent contextsthose that cannot equate Int and Char, for example. Below, we state the progress and preservation theorems and give a precise definition of consistency. In the next subsection, we formulate sufficient conditions for proving that a context is consistent. 


\subsection{Preservation and progress}

The preservation proof for $\mathrm{FC}_{2}$ is standard, relying on the usual regularity and substitution lemmas for the various judgement forms. For space reasons, we omit those definitions here and instead refer the reader to the appendices.

THEOREM 12 (Preservation). If $\Gamma \vdash e_{1}: \sigma$ and $e_{1} \rightsquigarrow e_{2}$ then $\Gamma \vdash e_{2}: \sigma$.

The progress theorem holds only for closed, consistent contexts. A context is closed if it does not contain any term variable bindings. We use the metavariable $\Sigma$ for closed contexts.

The definition of consistency and the canonical forms lemma (necessary to show the progress theorem) are both stated using the notions of uncoerced values and their types, value types. Value types include all types except those that are headed by a variable $a$ or type function/newtype $F$. Formally, we define values $v$ and value types $\tau$, with the following grammars:

$$
\begin{aligned}
& \tau \quad::=T|(\rightarrow)|\left(\sim_{\eta}\right)|\forall a: \kappa \cdot \varphi| \tau \varphi \\
& v \quad:=\lambda x: \sigma . e|\Lambda a: \kappa . e| \Lambda c: \varphi_{1} \sim \varphi_{2} . e \mid K \bar{\varphi} \bar{\rho}
\end{aligned}
$$

The canonical forms lemma tells us that the shape of a value is determined by its type: ${ }^{3}$

LEMMA 13 (Canonical Forms). Say $\Sigma \vdash v: \sigma$. Then $\sigma$ is a value type. Furthermore,
1. If $\sigma=\sigma_{1} \rightarrow \sigma_{2}$ then $v$ is $\lambda x: \sigma_{1}$.e or $K \bar{\varphi} \bar{\rho}$.
2. If $\sigma=\forall a: \kappa . \sigma^{\prime}$ then $v$ is $\Lambda$ a: $\kappa$.e or $K \bar{\varphi} \bar{\rho}$.
3. If $\sigma=\left(\varphi_{1} \sim \varphi_{2}\right) \Rightarrow \sigma^{\prime}$ then $v$ is $\Lambda c: \varphi_{1} \sim \varphi_{2}$. e or $K \bar{\varphi} \bar{\rho}$.
4. If $\sigma=T \bar{\varphi}_{1}$ then $v$ is $K \bar{\varphi} \bar{\rho}$.

In $\mathrm{FC}_{2}$, not all irreducible forms are values. Evaluation can also produce a coerced value of the form $v \triangleright \gamma$, which erases to a value when coercions are dropped. To prove the progress theorem, we must reason about what sort of coercion $\gamma$ could be so that we can appropriately apply the "push" rules in Figure 5. In the statement of the progress theorem, because $\gamma$ coerces the value $v$, we know (by ECOERCE and canonical forms) that $\gamma: \tau \sim \sigma$-the left type is a value type $\tau$. Consistency of the axiom set assures us that if $\sigma$ is also a value type, it must have the same head form.

DeFinition 14 (Consistency). A context $\Gamma$ is consistent if whenever $\Gamma \vdash \gamma: \tau_{1} \sim \tau_{2} \in \eta / \mathrm{T}$ it is the case that
1. If $\tau_{1}$ is $T \bar{\varphi}_{1}$ then $\tau_{2}$ is $T \bar{\varphi}_{2}$.
2. If $\tau_{1}$ is $(\rightarrow) \bar{\varphi}_{1}$ then $\tau_{2}$ is $(\rightarrow) \bar{\varphi}_{2}$.
3. If $\tau_{1}$ is $\left(\sim_{\eta}\right) \bar{\varphi}_{1}$ then $\tau_{2}$ is $\left(\sim_{\eta}\right) \bar{\varphi}_{2}$.
4. If $\tau_{1}$ is $\forall a: \kappa . \sigma_{1}$ then $\tau_{2}$ is $\forall a: \kappa . \sigma_{2}$.

Putting these observations together, we obtain:

THEOREM 15 (Progress). If $\Sigma$ is consistent and $\Sigma \vdash e_{1}: \sigma$ and $e_{1}$ is not a value $v$ or a coerced value $v \triangleright \gamma$, then there exists an $e_{2}$ such that $e_{1} \rightsquigarrow e_{2}$.

\subsection{Determining consistency}

Although the previous subsection gives a definition of when contexts are consistent, it does not provide any mechanism for determining whether a set of axioms leads to a consistent context.

This subsection defines sufficient conditions (written Good $\Gamma$ ) for establishing context consistency-these conditions are not the only way to show consistency (they are not necessary) but they are permissive enough to cover the axioms generated by compilation of type family declarations and newtype definitions.

\footnotetext{
${ }^{3}$ Note that all forms of value type must include partial applications of data constructors.
}

$$
\begin{gathered}
\hline \Gamma \vdash \varphi \rightsquigarrow \varphi^{\prime} \in \kappa \\
\Gamma \vdash a: \bar{\kappa} \rightarrow \eta / R \\
\frac{\Gamma \vdash \bar{\varphi} \rightsquigarrow \bar{\varphi}^{\prime} \in \bar{\kappa} / R}{\Gamma \vdash a \bar{\varphi} \rightsquigarrow a \bar{\varphi}^{\prime} \in \eta / R} \quad \text { RVAR } \\
H: \bar{\kappa} \rightarrow \eta \in \Gamma \\
\frac{\Gamma \vdash \bar{\varphi} \rightsquigarrow \bar{\varphi}^{\prime} \in \bar{\kappa} / R}{\Gamma \vdash H \bar{\varphi} \rightsquigarrow H \bar{\varphi}^{\prime} \in \eta / R} \quad \text { RCONST } \\
\frac{\Gamma, a: \kappa \vdash \sigma \rightsquigarrow \sigma^{\prime} \in \star / R}{\Gamma \vdash \forall a: \kappa . \sigma \rightsquigarrow \forall a: \kappa . \sigma^{\prime} \in \star / R} \quad \text { RALL } \\
H: \bar{\kappa}_{1} \rightarrow\left(\bar{\kappa}_{2} \rightarrow \eta\right) \in \Gamma \\
\Gamma \vdash \bar{\varphi}_{1} \rightsquigarrow \bar{\varphi}_{1}^{\prime} \in \bar{\kappa}_{1} / R \\
\Gamma \vdash \bar{\varphi}_{2} \rightsquigarrow \bar{\varphi}_{2}^{\prime} \in \bar{\kappa}_{2} / R \\
\frac{\Gamma \vdash c \bar{\psi}: H \bar{\varphi}_{1}^{\prime} \sim \sigma \in \bar{\kappa}_{2} \rightarrow \eta / R}{\Gamma \vdash H \bar{\varphi}_{1} \bar{\varphi}_{2} \rightsquigarrow \sigma \bar{\varphi}_{2}^{\prime} \in \eta / R} \quad \text { RRED } \\
\qquad \bar{\varphi}^{\prime} \in \bar{\kappa} / R
\end{gathered}
$$

$$
\begin{gathered}
\overline{\Gamma \vdash \cdot \rightsquigarrow \cdot \in \cdot / R} \quad \text { RNIL } \\
\Gamma \vdash \varphi \rightsquigarrow \varphi^{\prime} \in \eta / \min \left(R_{1}, R_{2}\right) \\
\frac{\Gamma \vdash \bar{\varphi} \rightsquigarrow \bar{\varphi}^{\prime} \in \bar{\kappa} / R_{1}}{\Gamma \vdash \varphi, \bar{\varphi} \rightsquigarrow \varphi^{\prime}, \bar{\varphi}^{\prime} \in \eta / R_{2}, \bar{\kappa} / R_{1}} \quad \text { RCONS }
\end{gathered}
$$

Figure 7. Type rewriting

As in the previous version of FC, we show consistency by (i) defining a rewrite system for types and (ii) proving that two types are joinable (share a common reduct) if and only if there is some coercion proof between those types. The rewrite system guarantees that value types preserve their head form throughout rewriting and therefore value types with different head forms can never be equated.

\subsubsection{Rewriting and joinability}

Figure 7 gives our rewrite relation, which is a variant of parallel reduction-it looks throughout the type, trying to fire reductions. The reduction of a type headed by a constant $H$ is implemented in rule RRED. If, after the arguments to $H$ have been reduced to $\bar{\varphi}^{\prime}$, there is some instantiation of an axiom such that $H$ applied to some prefix of the $\bar{\varphi}^{\prime}$ matches the left-hand side of the coercion, then the type may reduce to the right-hand-side type (RRED). Importantly, rewriting (very much like coercibility) takes roles into account: rewriting occurs at some role $R$, which specifies what axioms are available. For example, at role $T$ we can rewrite a newtype Age to its definition Int, but at role $C$, we cannot.

Notice that rules RCONST and RRED overlap. The term $H \bar{\varphi}$ may reduce using a matching axiom for $H$ (via rule RRED), but not necessarily (via RCONST). With rule RCONST, the rewrite relation is reflexive.

LEMMA 16. If $\Gamma \vdash \varphi: \kappa$ then $\Gamma \vdash \varphi \rightsquigarrow \varphi \in \kappa$.

The joinability relation, below, is simply reduction to a common reduct after zero or more steps.

DEFINITION 17 (Joinable). Two types are joinable if they share a common reduct. Define $\Gamma \vdash \varphi_{1} \Leftrightarrow \varphi_{2} \in \kappa$ if $\Gamma \vdash \varphi_{1} \rightsquigarrow^{*} \varphi \in \kappa$ and $\Gamma \vdash \varphi_{2} \rightsquigarrow^{*} \varphi \in \kappa$. 
Reflexivity of the rewrite relation is crucial for joinability of type applications with multiple arguments. Consider the axiom

$$
\mathrm{C}: \mathrm{F} \text { Int } \sim(\mathrm{F} \text { Int, } \mathrm{F} \text { Int }) / \mathrm{C}
$$

and the following two types:

$$
\begin{aligned}
& \varphi_{1}=\mathrm{T} \quad(\mathrm{F} \text { Int }) \quad(\mathrm{F} \text { Int, } \mathrm{F} \text { Int }) \\
& \varphi_{2}=\mathrm{T} \quad(\mathrm{F} \text { Int, } \mathrm{F} \text { Int) ( } \mathrm{F} \text { Int })
\end{aligned}
$$

We'd certainly like $\varphi_{1}$ and $\varphi_{2}$ to be reducible to a common reduct (after all, there exists a coercion term between them), but the first argument of $\mathrm{T}$ in $\varphi_{1}$ "lags behind" compared to the first argument of $\mathrm{T}$ in $\varphi_{2}$, whereas the second argument in $\varphi_{1}$ "advances ahead" compared to the second argument in $\varphi_{2}$. Reflexivity allows us to freeze the reduction of the second argument in $\varphi_{1}$ while reducing the first, and similarly for $\varphi_{2}$-which allows us to join the two types.

\subsubsection{Soundness and completeness of the rewrite relation}

We now give sufficient conditions on contexts which ensure that the rewrite relation of Figure 7 is sound and complete with respect to coercibility. Hence, these are sufficient conditions for consistency.

DEFINITION 18 (Good contexts). We have Good $\Gamma$ when the following conditions hold:

1. All axioms in $\Gamma$ are of the form $c: \Delta . F \bar{\varphi} \sim \psi / R$. Let $\bar{a}=\operatorname{dom}(\Delta)$ and $\bar{\kappa}$ be the corresponding kinds. For every well kinded $\bar{\psi}$ and result types $\bar{\varphi}^{\prime}$, such that $\Gamma \vdash(\bar{\varphi}[\bar{a} \mapsto \bar{\psi}]) \rightsquigarrow$ $\bar{\varphi}^{\prime} \in \bar{\kappa}^{\prime} / R$ it must be $\bar{\varphi}^{\prime}=\bar{\varphi}\left[\bar{a} \mapsto \bar{\psi}^{\prime}\right]$ for some $\bar{\psi}^{\prime}$ with $\Gamma \vdash \bar{\psi} \rightsquigarrow \bar{\psi}^{\prime} \in \bar{\kappa} / R$.

2. There is no overlap between axioms. For each $F \bar{\varphi}$ there exists at most one prefix $\bar{\varphi}_{1}$ of $\bar{\varphi}$ such that there exist $c, \psi$ and $\bar{\psi}$ where $\Gamma \vdash c \bar{\psi}: F \bar{\varphi}_{1} \sim \psi \in \kappa$. This $c$ is unique for every matching $F \bar{\varphi}_{1}$.

The first condition above restricts the declared arguments of type functions to behave like patterns. It merely states that all the reductions that can possibly happen when we substitute types for $\Delta$ do not involve reductions from $\bar{\varphi}$ but only reductions inside those substituted types. This condition is a generalized (and more lenient) form of the current GHC restrictions on type family declarations, which only allow value types or variables as $\bar{\varphi}$. The second condition is simply a strong non-overlapping condition.

Interestingly, the first condition on the arguments to type functions restricts the kind that a type function may have. For example, recall the axioms for $\mathrm{F}$ from Section 2.2:

$$
\begin{aligned}
& \operatorname{axF} 1: \mathrm{F} \text { Int } \sim \mathrm{Bool} / \mathrm{C} \\
& \operatorname{axF} 2: \mathrm{F} \text { Age } \sim \mathrm{Char} / \mathrm{C}
\end{aligned}
$$

For this to be a Good axiom set, the kind of $\mathrm{F}$ must be $\star / \mathrm{C} \rightarrow \star$ because the newtype Age is irreducible only in role C.

In the rest of this section, we sketch the proof that our conditions are sufficient for consistency. Soundness is a straightforward proof.

THEOREM 19 (Soundness). If if $\operatorname{Good} \Gamma$ and $\Gamma \vdash \varphi_{1} \rightsquigarrow \varphi_{2} \in$ $\kappa$ then there is some $\gamma$ such that $\Gamma \vdash \gamma: \varphi_{1} \sim \varphi_{2} \in \kappa$.

The three key lemmas of the completeness proof are that joinability is preserved under application and substitution and a local diamond property of rewriting.

LEMMA 20 (Application). If $\operatorname{Good} \Gamma$ and $\Gamma \vdash \varphi_{1} \Leftrightarrow \varphi_{1}^{\prime} \in$ $\left(\eta_{1} / R_{1} \rightarrow \eta_{2}\right) / R_{2}$ and $\Gamma \vdash \varphi_{2} \Leftrightarrow \varphi_{2}^{\prime} \in \eta_{1} / \min \left(R_{1}, R_{2}\right)$ then $\Gamma \vdash \varphi_{1} \varphi_{2} \Leftrightarrow \varphi_{1}^{\prime} \varphi_{2}^{\prime} \in \eta_{2} / R_{2}$.

THEOREM 21 (Local diamond property). If $\operatorname{Good} \Gamma$ and $\Gamma \vdash$ $\varphi \rightsquigarrow \varphi_{1} \in \kappa$ and $\Gamma \vdash \varphi \rightsquigarrow \varphi_{2} \in \kappa$ then there exists $a \varphi_{3}$ such that $\Gamma \vdash \varphi_{1} \rightsquigarrow \varphi_{3} \in \kappa$ and $\Gamma \vdash \varphi_{2} \rightsquigarrow \varphi_{3} \in \kappa$.
Lemma 22 (Substitution). If Good $\Gamma$ and $\Gamma, a: \kappa, \Delta \vdash \sigma \rightsquigarrow *$ $\sigma^{\prime} \in \kappa^{\prime}$ and $\Gamma \vdash \varphi \rightsquigarrow^{*} \varphi^{\prime} \in \kappa$, then there is some $\Gamma, \Delta \vdash$ $\sigma[a \mapsto \varphi] \Leftrightarrow \sigma^{\prime}\left[a \mapsto \varphi^{\prime}\right] \in \kappa^{\prime}$.

From these lemmas we see that joinability is complete.

Theorem 23 (Completeness). If $\operatorname{Good} \Gamma$ and $\Gamma \vdash \gamma: \varphi_{1} \sim$ $\varphi_{2} \in \kappa$ then $\Gamma \vdash \varphi_{1} \Leftrightarrow \varphi_{2} \in \kappa$.

Theorem 24 (Consistency). If Good $\Gamma$ then $\Gamma$ is consistent.

Proof Suppose $\Gamma \vdash \gamma: \tau_{1} \sim \tau_{2} \in \star / R$, where $\tau_{1}$ and $\tau_{2}$ are two value types. By completeness, we have that those two types are joinable, i.e. that there is some $\varphi$ such that $\Gamma \vdash \tau_{1} \rightsquigarrow^{*} \varphi \in \star / R$ and $\Gamma \vdash \tau_{2} \rightsquigarrow{ }^{*} \varphi \in \star / R$. However, by inversion on the rewriting relation, we see that it preserves the head forms of value types (since there exist no axioms for those by the first condition of Good $\Gamma$ ). Thus, $\tau_{1}$ and $\tau_{2}($ and $\varphi$ ) have the same head form.

A different novelty of our approach compared to previous work [Sulzmann et al. 2007] is that establishing the completeness theorem, and therefore type soundness, does not depend on strong normalization. Our definition of Good $\Gamma$ that (i) forces the declared type family arguments to behave like patterns, and (ii) imposes strong (stronger than the previous work) non-overlapping conditions, is sufficient to show completeness.

With this new approach, a programmer can be confident that, even in the presence of possibly non-terminating type functions, if the compiler can show that the program is well-typed then the program will not crash. Although type checking is undecidable in general without strong normalization, there may be many programs that the compiler can actually type check, and this approach shows that those programs are type sound.

\section{Compilation from source Haskell}

In the previous sections we have informally presented the translations of Haskell source features such as datatype, type family, and newt ype declarations into $\mathrm{FC}_{2}$. We summarize them here:

$$
\begin{array}{cc}
\text { data } T \Delta \text { where } \overline{K_{i}: \sigma} & \mapsto \\
T: \forall \Delta . \star, \overline{K_{i}: \Delta \cdot \sigma} & \\
\text { type family } F: \overline{\eta / C} \rightarrow \eta & \\
F: \overline{\eta / C} \rightarrow \eta & \mapsto \\
\text { type instance } \Delta \cdot F \bar{\varphi}=\psi & \\
c F: \Delta . F \bar{\varphi} \sim \psi \in \eta / C & \\
\text { newtype } \Delta . M \bar{a}=\mathrm{MkM} \varphi & \mapsto \\
M: \Delta . \star, \mathrm{MkM}: \Delta . M \bar{a} \sim \varphi \in \star / \mathrm{T} &
\end{array}
$$

In addition, uses of the data constructor of a newtype, both in terms and patterns, are translated to a use of the corresponding coercion in the obvious way. The important parts of these definitions are that (i) type families accept code arguments, (ii) type instances give rise to code equalities, and (iii) newtype definitions give rise to type equality axioms. The bindings generated in this way can be easily checked for well-formedness. If, in addition, the resulting context is Good (see Section 4.2) - the only possible problem is the potential to generate overlapping type instance declarations- then the context is consistent, which in turn guarantees type safety.

Notice that the source language features in this translation have been already annotated with their kinds. This is a reasonable assumption. Prior to type inference, which translates a source declaration to an $\mathrm{FC}_{2}$ binding, we must have determined the kinds involved in the declarations. For the purposes of this paper, we assume that the kinds are given-in practice they would be the output of a kind inference process, potentially guided by the user to disambiguate role information and higher-order kinds. 
Newtype deriving Generative type abstraction is achieved in Haskell using the newtype deriving mechanism, which allows type classes to be automatically lifted to new types. For instance, we may write

$$
\text { newtype Age = MkAge Int deriving Eq }
$$

The type class Eq a is a standard class in Haskell (signature, in ML terminology) that defines one method, eq $:: \mathrm{a} \rightarrow \mathrm{a} \rightarrow$ Bool. For the type checker, a type class is nothing but a record type containing the method eq. The deriving line automatically generates an implementation of Eq Age from a pre-existing instance Eq Int. This can be done by simply applying a coercion $\mathrm{Eq}$ Int $\sim \mathrm{Eq}$ Age to the old record. It is straightforward to construct this coercion: lift the Age $\sim$ Int axiom from the newtype definition over the Eq constructor and apply symmetry. Importantly, this lifting is safe because $\mathrm{Eq}$ has a parametric kind of the form $\star / T \rightarrow \star$. However, if a type class has a indexed kind of the form $\star / C \rightarrow \star$, newtype deriving is no longer sound. For example

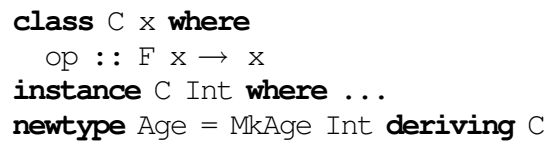

It is unsound to lift the $C$ Int implementation to a $C$ Age, precisely because $x$ has role $C$. The documented GHC bug mentioned earlier arises precisely as the result of such an unsound lifting over a non-parametric type class.

\section{Discussion}

\subsection{Relaxing decomposition}

Recall the coercion decomposition rule, CNTHT, from Figure 4. This rule allows us to deconstruct an equality of the form $\Gamma \vdash \gamma$ : $T \bar{\varphi} \sim T \bar{\psi} \in \eta / T$. In effect, it asserts that data constructors are injective. The rule is important because it is used in the operational semantics to ensure subject reduction. However, the decomposition rule may be somewhat restrictive for some Haskell source programs. Consider the following:

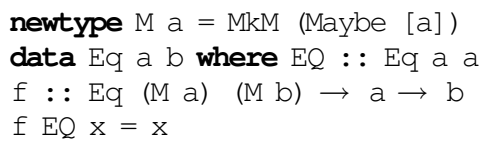

Pattern matching against the $\mathrm{E} Q$ constructor introduces a coercion between $\mathrm{M}$ a and $\mathrm{M} b$, which cannot be decomposed using the CNTHT rule to obtain $a \sim b$, so this program cannot be typed. Nevertheless, we know that $\mathrm{M}$ is injective, because $\mathrm{M}$ a is defined to be equal to Maybe [a], which is clearly injective.

On the other hand, the following newtype is not injective.

type instance $\mathrm{G}$ a $=$ Char

newtype $\mathrm{N}$ a $=\mathrm{MkN}$ ( $\mathrm{G}$ a)

Here, it is possible to derive $\Gamma \vdash \gamma: \mathrm{N}$ Int $\sim \mathrm{N}$ Char $\in \star / \mathrm{T}$, using the axiom for $\mathrm{G}$, even though Int is not coercible to Char.

It turns out that newtype definitions are always injective with respect to code equality, but they might not be injective with respect to type equality (as illustrated by the two examples above). Thus it would be sound and potentially useful (but not necessary for type soundness) to introduce yet another decomposition rule for newtype definitions that takes advantage of injectivity with respect to codes (we use metavariable $N$ newtypes):

$$
\begin{array}{cl}
\Gamma \vdash \gamma: N \bar{\varphi} \sim N \bar{\psi} \in \eta / \mathrm{C} & \\
N: \bar{\kappa} \rightarrow \eta \in \Gamma & \\
\eta^{\prime} / R^{\prime}=n t h k \bar{\kappa} & \text { CNTHN } \\
\Gamma \vdash \mathbf{n t h} k \gamma: \text { nth } k \bar{\varphi} \sim \text { nth } k \bar{\psi} \in \eta^{\prime} / R &
\end{array}
$$

The only subtle part of this rule is that $R$ is not related to $R^{\prime}$, since the equality $\Gamma \vdash \gamma: N \bar{\varphi} \sim N \bar{\psi} \in \eta / \mathrm{C}$ is a C-equality (and $\left.\min \left(\mathrm{C}, R^{\prime}\right)=\mathrm{C}\right)$.

Arguably, decomposition for injective type functions is also desirable, were we able to effectively specify and check that property.

\subsection{Other technical differences of $\mathrm{FC}_{2}$ from System FC}

The intermediate language $\mathrm{FC}_{2}$ described in this paper is a significant modification of System FC [Sulzmann et al. 2007] due to the introduction of codes. However, $\mathrm{FC}_{2}$ also makes a number of technical simplifications:

- The original System FC presentation includes coercion kinds, $\sigma_{1} \sim \sigma_{2}$. The original coercion language includes three additional constructs, one to coerce coercions, and two more to decompose coercion kinds. By treating $\left(\varphi_{1} \sim \varphi_{2}\right) \Rightarrow \varphi_{3}$ as the application of a constructor $\left(\sim_{\eta}\right)$ we no longer need any of these constructs in the operational semantics, nor have we identified any uses of these constructs that are not encodable.

- The operational semantics rules of $\mathrm{FC}_{2}$ in Figure 5 not only use simpler coercion constructs, but are also expressed without need for substitutions, contrary to their original FC versions.

- $\mathrm{FC}_{2}$ replaces the FC coercions left and right, which decomposed arbitrary type applications, by nth, which decomposes only the application of a datatype constructor. This allows us to lift a tiresome restriction in in System FC, namely that type functions were required to be saturated. Type family saturation was necessary in FC, in order to prevent the decomposition of equalities as $\mathrm{F} a \sim$ Maybe $[a]$ via left or right. Allowing unsaturated functions increases the expressiveness of $\mathrm{FC}_{2}$, because we can now abstract over type functoins, and also opens new directions for future research on type inference in the presence of unsaturated type functions.

- However, using nth instead of left and right does carry a small price. Generalizing the example from Section 6.1, should this program be well typed?

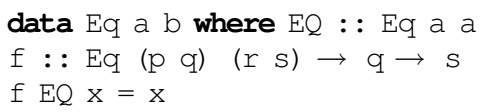

To type it we must decompose a proof that $p q \sim r s$ to get a proof that $q \sim s$, which right could do, but nth cannot.

Some other differences are presentational:

- System FC used a common syntax for types and coercions, which is a convenient pun, but has turned out to be more confusing than helpful. In $\mathrm{FC}_{2}$ we use a distinct syntax for types and coercions (Figure 1).

- In $\mathrm{FC}_{2}$ we define top-level axiom schemes $c: \Delta . \varphi_{1} \sim \varphi_{2} / R$ directly, and fully instantiate them at every occurrence with the form $c \bar{\gamma}$ (Figure 1). System FC instead defined a toplevel axiom scheme as an equality between polytypes, thus $c: \forall \Delta . \varphi_{1} \sim \forall \Delta . \varphi_{2}$. Here again $\mathrm{FC}$ is confusing (but not wrong) so in $\mathrm{FC}_{2}$ we opt for telling the story more directly, albeit with slightly more syntax. Moreover the kinding rules for $\forall$ (PALL and CALL) can insist that the body of the forall has kind $\star$ as is conventional. 
- Using telescopes in the treatment of datatypes simplifies the operational semantics rules but is also (only slightly) more expressive: The types of data constructors do not have to have their quantified variables preceding their coercion and term arguments. Instead, telescopes allow arbitrary interleavings.

\section{Related work}

Previous work on System FC [Sulzmann et al. 2007] discusses a significant amount of related work, in typed languages with explicit proof witnesses [Licata and Harper 2005; Shao et al. 2005], or in calculi that support coercions [Breazu-Tannen et al. 1991]. Below, we present related work in generativity and abstraction, typeindexed constructs and the separation between codes and types.

Generativity, abstraction, and module systems Generativity and abstraction has been studied extensively in the context of ML module systems [Milner et al. 1997]. Russo shows how generativity in module systems is connected to existential quantification [Russo 1999] and Dreyer [2005] has studied this connection in the presence of recursive modules. Montagu and Rémy [2009] refine this connection by introducing "open" existential types. Rossberg [2008] uses flexible generativity to explain ML-style module sealing.

Type abstraction can be understood in terms of dynamic name generation [Rossberg 2003; Vytiniotis et al. 2005], which can reestablish abstraction properties in languages with dynamic type analysis. Neis et al. [2009] prove a parametricity theorem in this setting. In addition, they use a translation from polymorphism to generative types to establish the parametric behavior of certain functions although they work in a non-parametric language.

Although many of these languages support type generativity and non-parametric features, they do not exhibit the soundness problems described in the paper, mainly due to the absence of typelevel type dispatching. Nevertheless, the techniques developed in the aforementioned related work would be valuable in the formal study of the parametricity properties of $\mathrm{FC}_{2}$.

Type-indexed types Although many systems for generic programming support dynamic computation based on types, very few systems allow the structure of types to be destructed to produce other types. However, such facility is often necessary to describe the type of generic programs. For example, Harper and Morrisett include a Typerec operator to their typed intermediate language $\lambda_{i}^{M L}$ [Harper and Morrisett 1995], to describe type-directed optimizations. (They credit NuPRL's mechanism of "Universe Elimination" in NuPRL as the inspiration for this operation [Constable 1982; Constable and Zlatin 1984].)

To support generic programming in source languages, Hinze, Jeuring and Löh added Type-Indexed Datatypes [Hinze et al. 2002] to the Generic Haskell front end. In later work, Chakravarty et al. [2005b] introduced associated data families in GHC, which are type-indexed datatypes associated with type class instances. Extending this work, they later introduced associated type synonyms [Chakravarty et al. 2005a], which are proper type-level functions with instances associated with type class instances. Currently, the source language of GHC also supports standalone type-level type functions, often referred to as indexed type families [Kiselyov et al. 2010; Schrijvers et al. 2008], a feature that we have extensively used in our presentation.

Codes, types, and interpretations Our distinction between codes and types - and our terminology — is inspired by similar notions in intuitionistic type theory [Benke et al. 2003; Dybjer 2000; MartinLöf 1975]. There, types (sets) are constructed as the recursive interpretation of codes, which inhabit inductively constructed code universes. A newtype definition can be viewed as giving rise to a new code, inhabiting an open universe of codes, and whose interpretation coincides with the interpretation of its definition.

Languages based on dependent type theory, such as Agda [Bove et al. 2009] or Coq [The Coq Team], naturally offer type-level computation to construct types, but they allow elimination of codes only, not types. Therefore, they do not exhibit the same soundness problem, as the expressiveness of these languages can readily enforce the distinction between types and codes. The disadvantage is the extra programming verbosity of explicit definitions and interpretations of codes. To better support generic programming, the dependently-typed language Epigram [Chapman et al. 2010] identifies types with their code universes.

The LX language [Crary and Weirich 1999] also uses universe constructions to solve problems with type-directed compilation. When the type translation in a compiler pass is not the identity then type dispatch must be compiled to code dispatch (so the generated code can dispatch on source types instead of target types). The interpretation of codes is then the type translation. To support universes, LX includes datakinds (for codes) and primitive recursive functions over datakinds (for their interpretation at types). In LX, source types Age and Int would be mapped to definable codes AgeCode and IntCode, and would be accompanied by an interpretation function such that interp (AgeCode) equals Int and interp (IntCode) equals Int. Therefore, the problem with generativity would not show up in that context. If one wanted to solve the problem in this paper along the LX lines, one would have to translate source Haskell types to void types that stand in as codes and handle the interp () function as any other type function. This function, as well as interpreting the codes as types, would have to be accompanied with suitable congruence axioms, like interp $(\mathrm{T} t) \sim \mathrm{T}$ (interp $(t))$. Explicitly introducing these axioms means that coercions would be significantly more verbose. Our system dispenses with an explicit interp () function by conveniently using the roles in the judgements to determine whether we wish to derive an equality between codes or between their interpretations.

\section{Conclusions and future work}

In this paper we have identified a problem for the safe interaction of flexible type generativity and type-level computation. We have proposed a solution that distinguishes between indexed and parametric type contexts, by extending the language of kinds, and formalized the solution in the $\mathrm{FC}_{2}$ language. We have several avenues for future research in mind, which we outline below.

Source language technology We would like to work on ways to expose the $\mathrm{FC}_{2}$ expressive features to programmers. Specific directions are: enriching the kind declarations with the ability to declare parametric or indexed type-level constructs, introducing type family injectivity annotations, extending kind inference with roles, and extending type inference to support unsaturated functions using the more sophisticated kinds.

Enriching the universes of codes with terms We are currently working on enriching the universe of codes with constants or functions drawn from the term syntax, such as data constructors, in order to enable direct dependently-typed programming in Haskell.

More roles Lemma 6 asserts that the equivalence classes induced by $\mathrm{T}$-equality are refined by $\mathrm{C}$-equality. However, our approach readily extends to arbitrary lattices of roles with gradually more refined equivalence classes as we move down the $\preceq$ relation.

Consider, for example, a lattice with new roles $C_{1}$ and $C_{2}$, each lying between $C$ and $T$, but incomparable with each other. These two roles model partial knowledge of newtype equalities. For example, perhaps $C_{1}$ identifies Age and Int but distinguishes 
a different newtype Moo from its definition Bool. Conversely, $\mathrm{C}_{2}$ can identify Moo and Bool and distinguish Age and Int. These more precise roles could be used to give more precise types to nonparametric functions by identifying exactly which newtype equivalences they do and do not respect. However, we have not yet explored the practical implications of this precision.

Furthermore, one could explore adding a role above T. Type functions with kind $\star / T \rightarrow \star$, are not necessarily parametric in $\mathrm{FC}_{2}$. For example, because $\mathrm{G}$ below does not match newtypes it may be assigned either kind $\star / \mathrm{T} \rightarrow \star$ or $\star / \mathrm{C} \rightarrow \star$.

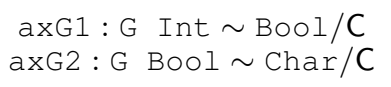

To distinguish truly parametric functions from those like $G$, we could add a role $P$, induced by the relation that equates all types of the same kind. As this equivalence is coarser than $T$, it could not (and should not) participate in coercions. However, this new role would provide a yet more descriptive kind for type functions.

\section{Acknowledgements}

We thank Brent Yorgey and the attendees of the Type System Wrestling sessions at MSR Cambridge for many useful discussions. We wrote this paper using the Ott tool. This work was partially supported by grants from DARPA (CSSG) and NSF (0910786) and a Sloan Foundation fellowship.

\section{References}

M. Benke, P. Dybjer, and P. Jansson. Universes for generic programs and proofs in dependent type theory. Nordic J. of Computing, 10(4):265289, 2003. ISSN 1236-6064.

A. Bove, P. Dybjer, and U. Norell. A brief overview of agda - a functional language with dependent types. In TPHOLs '09: Proceedings of the 22nd International Conference on Theorem Proving in Higher Order Logics, pages 73-78, Berlin, Heidelberg, 2009. Springer-Verlag.

V. Breazu-Tannen, T. Coquand, C. A. Gunter, and A. Scedrov. Inheritance as implicit coercion. Information and Computation, 93:172-221, 1991.

M. M. T. Chakravarty, G. Keller, and S. Peyton Jones. Associated type synonyms. In ICFP '05: Proceedings of the Tenth ACM SIGPLAN International Conference on Functional Programming, pages 241-253, New York, NY, USA, 2005a. ACM.

M. M. T. Chakravarty, G. Keller, S. Peyton Jones, and S. Marlow. Associated types with class. SIGPLAN Not., 40(1):1-13, 2005b. ISSN 0362-1340.

J. Chapman, P. Évariste Dagand, C. McBride, and P. Morris. The gentle art of levitation. In Proceedings of the Fifteenth ACM SIGPLAN International Conference on Functional Programming (ICFP '10), Baltimore, MD, USA, September 2010. To appear.

J. Cheney and R. Hinze. First-class phantom types. CUCIS TR2003-1901, Cornell University, 2003.

R. L. Constable. Intensional analysis of functions and types. Technical Report CSR-118-82, Department of Computer Science, University of Edinburgh, June 1982.

R. L. Constable and D. R. Zlatin. The type theory of PL/CV3. ACM Transactions on Programming Languages and Systems, 6(1):94-117, Jan. 1984.

K. Crary and S. Weirich. Flexible type analysis. In Proceedings of the Fourth ACM SIGPLAN International Conference on Functional Programming, pages 233-248, Paris, France, Sept. 1999.

N. G. de Bruijn. Telescopic mappings in typed lambda calculus. Inf. Comput., 91(2):189-204, 1991. ISSN 0890-5401.

D. Dreyer. Recursive type generativity. In ICFP '05: Proceedings of the Tenth ACM SIGPLAN International Conference on Functional Programming, pages 41-53, New York, NY, USA, 2005. ACM.

P. Dybjer. A general formulation of simultaneous inductive-recursive definitions in type theory. Journal of Symbolic Logic, 65(2):525-549, 2000.
C. V. Hall, K. Hammond, S. L. Peyton Jones, and P. L. Wadler. Type classes in Haskell. ACM Transactions on Programming Languages and Systems, 18(2):109-138, Mar. 1996.

R. Harper and G. Morrisett. Compiling polymorphism using intensional type analysis. In POPL '95: Proceedings of the 22nd ACM SIGPLANSIGACT symposium on Principles of programming languages, pages 130-141, New York, NY, USA, 1995. ACM. ISBN 0-89791-692-1.

R. Hinze, J. Jeuring, and A. Löh. Type-indexed data types. In B. M. Eerke Boiten, editor, Proceedings of the Sixth International Conference on Mathematics of Program Construction (MPC 2002), pages 148-174, Dagstuhl, Germany, July 2002.

O. Kiselyov, S. Peyton Jones, and C. Shan. Fun with type functions. Springer, 2010.

D. R. Licata and R. Harper. A formulation of dependent ML with explicit equality proofs. Technical Report CMU-CS-05-178, Carnegie Mellon University Department of Computer Science, 2005.

P. Martin-Löf. An intuitionistic theory of types: Predicative part. In Proceedings of the Logic Colloquium, 1973, volume 80 of Studies in Logic and the Foundations of Mathematics, pages 73-118. North-Holland, 1975.

R. Milner, M. Tofte, and D. Macqueen. The Definition of Standard ML. MIT Press, Cambridge, MA, USA, 1997. ISBN 0262631814.

B. Montagu and D. Rémy. Modeling abstract types in modules with open existential types. In POPL '09: Proceedings of the 36th annual ACM SIGPLAN-SIGACT Symposium on Principles of Programming Languages, pages 354-365, New York, NY, USA, 2009. ACM.

G. Neis, D. Dreyer, and A. Rossberg. Non-parametric parametricity. In ICFP '09: Proceedings of the 14th ACM SIGPLAN International Conference on Functional Programming, pages 135-148, New York, NY, USA, 2009. ACM. ISBN 978-1-60558-332-7.

S. Peyton Jones et al. The Haskell 98 language and libraries: The revised report. Journal of Functional Programming, 13(1):0-255, Jan 2003. http: //www.haskell.org/definition/.

S. L. Peyton Jones, D. Vytiniotis, S. Weirich, and G. Washburn. Simple unification-based type inference for GADTs. In International Conference on Functional Programming (ICFP), Portland, OR, USA, Sept. 2006.

B. C. Pierce, editor. Advanced Topics in Types and Programming Languages. MIT Press, 2005.

A. Rossberg. Dynamic translucency with abstraction kinds and higher-order coercions. Electr. Notes Theor. Comput. Sci., 218:313-336, 2008.

A. Rossberg. Generativity and dynamic opacity for abstract types. In PPDP '03: Proceedings of the 5th ACM SIGPLAN International Conference on Principles and Practice of Declaritive Programming, pages 241-252, New York, NY, USA, 2003. ACM. ISBN 1-58113-705-2.

C. V. Russo. Non-dependent types for Standard ML modules. In PPDP '99: Proceedings of the International Conference PPDP'99 on Principles and Practice of Declarative Programming, pages 80-97, London, UK, 1999. Springer-Verlag. ISBN 3-540-66540-4.

T. Schrijvers, S. Peyton Jones, M. Chakravarty, and M. Sulzmann. Type checking with open type functions. In ICFP '08: Proceeding of the 13th ACM SIGPLAN international conference on Functional programming, pages 51-62, New York, NY, USA, 2008. ACM.

Z. Shao, V. Trifonov, B. Saha, and N. Papaspyrou. A type system for certified binaries. ACM Trans. Program. Lang. Syst., 27(1):1-45, 2005.

M. Sulzmann, M. M. T. Chakravarty, S. Peyton Jones, and K. Donnelly. System F with type equality coercions. In TLDI '07: Proceedings of the 2007 ACM SIGPLAN International Workshop on Types in Languages Design and Implementation, pages 53-66, New York, NY, USA, 2007. ACM.

The Coq Team. Coq. URL http: / / coq. inria. fr.

D. Vytiniotis, G. Washburn, and S. Weirich. An open and shut typecase. In ACM SIGPLAN Workshop in Types in Language Design and Implementation, Long Beach, CA, USA, Jan. 2005.

H. Xi, C. Chen, and G. Chen. Guarded recursive datatype constructors. In POPL, pages 224-235, 2003. 


\section{A. Additional specification}

For space reasons, the following definitions and rules did not appear in the main text of the paper.

DEFINITION 25. We define $\forall \Delta . \sigma$ by induction on $\Delta$ as follows:

$$
\begin{array}{ll}
\forall \cdot \sigma & =\sigma \\
\forall(a: \kappa, \Delta) . \sigma & =\forall a: \kappa \cdot(\forall \Delta . \sigma)
\end{array}
$$

DEFINITION 26 (Multilifting Operation). Define the multiple lifting operation, written $\varphi[\bar{a} \mapsto \bar{\gamma}]$, by induction on $\varphi$.

$$
\begin{array}{lll}
a[\bar{a} \mapsto \bar{\gamma}] & =\gamma_{i} \text { when } a_{i} \text { in } \bar{a} \\
b[\bar{a} \mapsto \bar{\gamma}] & =b \quad \text { when } b \notin \bar{a} \\
H[\bar{a} \mapsto \bar{\gamma}] & =H \\
(\varphi \psi)[\bar{a} \mapsto \bar{\gamma}] & =(\varphi[\bar{a} \mapsto \bar{\gamma}])(\psi[\bar{a} \mapsto \bar{\gamma}]) \\
(\forall b: \kappa . \sigma)[\bar{a} \mapsto \bar{\gamma}] & =\forall b: \kappa \cdot(\sigma[\bar{a} \mapsto \bar{\gamma}])
\end{array}
$$

$\Gamma \vdash\{b n d\}$

$$
\begin{aligned}
& \overline{\Gamma \vdash\{a: \kappa\}} \quad \text { BTVAR } \\
& \overline{\Gamma \vdash\{(\rightarrow): \star / T \rightarrow \star / T \rightarrow \star\}} \quad \text { BARR } \\
& \overline{\Gamma \vdash\left\{\left(\sim_{\eta}\right): \eta / \mathrm{C} \rightarrow \eta / \mathrm{C} \rightarrow \star / \mathrm{T} \rightarrow \star\right\}} \quad \text { BCOERCE } \\
& \overline{\Gamma \vdash\{T: \eta\}} \quad \text { BCONST } \\
& \overline{\Gamma \vdash\{F: \eta\}} \quad \text { BFUN } \\
& \frac{\Gamma, \Delta \vdash \varphi_{1}: \eta / R \quad \Gamma, \Delta \vdash \varphi_{2}: \eta / R}{\Gamma \vdash\left\{c: \Delta . \varphi_{1} \sim \varphi_{2} / R\right\}} \quad \text { BCVAR } \\
& \frac{\Gamma \vdash \sigma: \star / \mathrm{T}}{\Gamma \vdash\{x: \sigma\}} \quad \text { BEVAR } \\
& \frac{\Gamma \vdash \sigma: \star / \mathrm{T}}{\Gamma \vdash\{K: \sigma\}} \quad \text { BDATACON }
\end{aligned}
$$

$\vdash \Gamma$

$e \rightsquigarrow e^{\prime}$

$$
\begin{array}{r}
\qquad \quad \text { GEMPTy } \\
\frac{\text { dom bnd\# dom } \Gamma \quad \Gamma \vdash\{b n d\}}{\vdash \Gamma, \text { bnd }} \text { GCONS }
\end{array}
$$

$$
\begin{aligned}
& \frac{\gamma_{0}=\operatorname{sym}(\mathbf{n t h} 0 \gamma) \quad \gamma_{1}=\mathbf{n t h} 1 \gamma}{\left(\left(\lambda x: \sigma_{1} \cdot e_{1}\right) \triangleright \gamma\right) e_{2} \rightsquigarrow\left(\lambda x: \sigma_{1} \cdot\left(e_{1} \triangleright \gamma_{1}\right)\right)\left(e_{2} \triangleright \gamma_{0}\right)} \\
& \overline{\left(\lambda x: \sigma . e_{1}\right) e_{2} \rightsquigarrow e_{1}\left[x \mapsto e_{2}\right]} \quad \text { SSBETA } \\
& \frac{e_{1} \rightsquigarrow e_{1}^{\prime}}{e_{1} e_{2} \rightsquigarrow e_{1}^{\prime} e_{2}} \quad \text { SSEAPP } \\
& \frac{\Sigma \vdash \gamma: \forall a: \kappa . \sigma_{1} \sim \forall a: \kappa . \sigma_{2} \in \star / \mathrm{T}}{((\Lambda a: \kappa . e) \triangleright \gamma) \varphi \rightsquigarrow(\Lambda a: \kappa .(e \triangleright \gamma @ a)) \varphi} \quad \text { SSTPUSH } \\
& \overline{(\Lambda a: \kappa . e) \varphi \rightsquigarrow e[a \mapsto \varphi]} \quad \text { SSTBETA } \\
& \frac{e_{1} \rightsquigarrow e_{1}^{\prime}}{e_{1} \varphi \rightsquigarrow e_{1}^{\prime} \varphi} \quad \text { SSTAPP } \\
& \Sigma \vdash \gamma:\left(\sim_{\eta}\right) \varphi_{1} \varphi_{2} \sigma \sim\left(\sim_{\eta}\right) \varphi_{1}^{\prime} \varphi_{2}^{\prime} \sigma^{\prime} \in \star / \mathrm{T} \\
& \frac{\gamma_{0}=\mathbf{n t h} 0 \gamma \quad \gamma_{1}=\mathbf{s y m}(\text { nth } 1 \gamma) \quad \gamma_{2}=\text { nth } 2 \gamma}{\left(\left(\Lambda c: \varphi_{1} \sim \varphi_{2} . e\right) \triangleright \gamma\right) \gamma^{\prime} \rightsquigarrow\left(\Lambda c: \varphi_{1} \sim \varphi_{2} .\left(e \triangleright \gamma_{2}\right)\right)\left(\gamma_{0} ; \gamma^{\prime} ; \gamma_{1}\right)} \quad \text { SSCPUSH }
\end{aligned}
$$




$$
\begin{aligned}
& \overline{\left(\Lambda c: \varphi_{1} \sim \varphi_{2} . e\right) \gamma \rightsquigarrow e[c \mapsto \gamma]} \quad \text { SSCBETA } \\
& \frac{e_{1} \rightsquigarrow e_{1}^{\prime}}{e_{1} \gamma \rightsquigarrow e_{1}^{\prime} \gamma} \quad \text { SSCAPP } \\
& \overline{\left(v \triangleright \gamma_{1}\right) \triangleright \gamma_{2} \rightsquigarrow v \triangleright\left(\gamma_{1} ; \gamma_{2}\right)} \\
& \frac{e \rightsquigarrow e^{\prime}}{e \triangleright \gamma \rightsquigarrow e^{\prime} \triangleright \gamma} \quad \text { SSCOERCE } \\
& \Sigma \vdash \gamma: T \bar{\varphi} \sim T \bar{\varphi}^{\prime} \in \star / \mathrm{T} \\
& K: \forall \bar{a}: \bar{\kappa} \cdot \sigma^{\prime} \in \Sigma \\
& \overline{\operatorname{case}_{\sigma}(K \bar{\varphi} \bar{\rho}) \triangleright \gamma \text { of brs } \rightsquigarrow \operatorname{case}_{\sigma} K \bar{\varphi}^{\prime}\left(\bar{\rho} \triangleright \sigma^{\prime}[\bar{a} \mapsto n t h \gamma]\right) \text { of brs }} \\
& \frac{1 \leq j \leq n}{\text { case }_{\sigma}\left(K_{j} \bar{\varphi} \bar{\rho}\right) \text { of }{\overline{K_{i} \Rightarrow e_{i}}}^{i \in 1 . . n} \rightsquigarrow e_{j} \bar{\rho}} \quad \text { SSCASEMATCH } \\
& \frac{e \rightsquigarrow e^{\prime}}{\text { case }_{\sigma} e \text { of brs } \rightsquigarrow \text { case }_{\sigma} e^{\prime} \text { of brs }} \quad \text { SSCASE }
\end{aligned}
$$

\section{B. Additional lemmas and proofs}

\section{B.1 Preservation and progress}

We state additional basic properties of our judgements below. Proofs of these properties are by straightforward induction.

LEMMA 27 (Type regularity). If $\Gamma \vdash e: \sigma$ then $\Gamma \vdash \sigma: \star / \top$ and $\vdash \Gamma$.

LEMMA 28 (Type substitution). Say $\Gamma_{1} \vdash \varphi^{\prime}: \kappa^{\prime}$.

1. If $\Gamma_{1}, a: \kappa^{\prime}, \Gamma_{2} \vdash \varphi: \kappa$ then $\Gamma_{1},\left(\Gamma_{2}\left[a \mapsto \varphi^{\prime}\right]\right) \vdash \varphi\left[a \mapsto \varphi^{\prime}\right]: \kappa$

2. If $\Gamma_{1}, a: \kappa^{\prime}, \Gamma_{2} \vdash \bar{\varphi}: \bar{\kappa}$ then $\Gamma_{1},\left(\Gamma_{2}\left[a \mapsto \varphi^{\prime}\right]\right) \vdash \bar{\varphi}\left[a \mapsto \varphi^{\prime}\right]: \bar{\kappa}$

3. If $\Gamma_{1}, a: \kappa^{\prime}, \Gamma_{2} \vdash \gamma: \varphi_{1} \sim \varphi_{2} \in \kappa$ then $\Gamma_{1},\left(\Gamma_{2}\left[a \mapsto \varphi^{\prime}\right]\right) \vdash \gamma\left[a \mapsto \varphi^{\prime}\right]: \varphi_{1}\left[a \mapsto \varphi^{\prime}\right] \sim \varphi_{2}\left[a \mapsto \varphi^{\prime}\right] \in \kappa$.

4. If $\Gamma_{1}, a: \kappa^{\prime}, \Gamma_{2} \vdash \bar{\gamma}: \bar{\varphi}_{1} \sim \bar{\varphi}_{2} \in \bar{\kappa}$ then $\Gamma_{1},\left(\Gamma_{2}\left[a \mapsto \varphi^{\prime}\right]\right) \vdash \bar{\gamma}\left[a \mapsto \varphi^{\prime}\right]: \bar{\varphi}_{1}\left[a \mapsto \varphi^{\prime}\right] \sim \bar{\varphi}_{2}\left[a \mapsto \varphi^{\prime}\right] \in \bar{\kappa}$.

5. If $\Gamma_{1}, a: \kappa^{\prime}, \Gamma_{2} \vdash e: \sigma$ then $\Gamma_{1},\left(\Gamma_{2}\left[a \mapsto \varphi^{\prime}\right]\right) \vdash e\left[a \mapsto \varphi^{\prime}\right]: \sigma\left[a \mapsto \varphi^{\prime}\right]$

LEMMA 29 (Coercion substitution). Say $\Gamma_{1} \vdash \gamma: \varphi_{1} \sim \varphi_{2} \in \eta / R$.

1. If $\Gamma_{1}, c: \varphi_{1} \sim \varphi_{2} / R, \Gamma_{2} \vdash \gamma^{\prime}: \psi_{1} \sim \underline{\psi}_{2} \in \kappa^{\prime}$ then $\Gamma_{1}, \Gamma_{2} \vdash \gamma^{\prime}[c \mapsto \gamma]: \psi_{1} \sim \psi_{2} \in \kappa^{\prime}$

2. If $\Gamma_{1}, c: \varphi_{1} \sim \varphi_{2} / R, \Gamma_{2} \vdash \bar{\gamma}: \bar{\psi}_{1} \sim \bar{\psi}_{2} \in \bar{\kappa}$ then $\Gamma_{1}, \Gamma_{2} \vdash \bar{\gamma}[c \mapsto \gamma]: \bar{\psi}_{1} \sim \bar{\psi}_{2} \in \bar{\kappa}$

3. If $\Gamma_{1}, c: \varphi_{1} \sim \varphi_{2} / R, \Gamma_{2} \vdash e: \sigma$ then $\Gamma_{1}, \Gamma_{2} \vdash e[c \mapsto \gamma]: \sigma$

LEMma 30 (Term substitution). Say $\Gamma_{1} \vdash e^{\prime}: \sigma^{\prime}$. If $\Gamma_{1}, x: \sigma^{\prime}, \Gamma_{2} \vdash e: \sigma$, then $\Gamma_{1}, \Gamma_{2} \vdash e\left[x \mapsto e^{\prime}\right]: \sigma$.

LEMma 31 (Multilifting). If $\Gamma, \Delta \vdash \sigma: \kappa$ and $\Gamma \vdash \gamma_{i}: \varphi_{i} \sim \varphi_{i}^{\prime} \in \kappa_{i}$ for each $a_{i}: \kappa_{i}$ in $\Delta$, then $\Gamma \vdash \sigma[\bar{a} \mapsto \bar{\gamma}]: \sigma[\bar{a} \mapsto \bar{\varphi}] \sim \sigma[\bar{a} \mapsto$ $\left.\bar{\varphi}^{\prime}\right] \in \kappa$

Next we sketch the proofs of the progress and preservation theorems.

Proof OF THEOREM 12 (Preservation): If $\Gamma \vdash e_{1}: \sigma$ and $e_{1} \rightsquigarrow e_{2}$ then $\Gamma \vdash e_{2}: \sigma$.

Proof by induction on $e_{1} \rightsquigarrow e_{2}$.

- Case SSBETA, SSTBETA, SSCBETA: Application of the appropriate substitution lemma.

- Case SSCASEMATCH: By inversion $\Gamma \vdash K_{j} \bar{\varphi} \bar{\rho}: T \bar{\varphi}$ and $K_{j}: \forall \Delta . \psi_{j} \in \Gamma$ and $\psi_{j}[\bar{a} \mapsto \bar{\varphi}]=\forall \Theta_{j} . T \bar{\varphi}$ and $\Gamma \vdash e_{j}: \forall \Theta_{j} . \sigma$. Further inversion gives $\Gamma \vdash \bar{\rho}: \Theta_{j}$ By repeated use of the application rule, $\Gamma \vdash e_{j} \bar{\rho}: \sigma$.

- Case SSPush: By inversion $\sigma=\sigma_{2}^{\prime}$ and $\Gamma \vdash \gamma:\left(\sigma_{1} \rightarrow \sigma_{1}^{\prime}\right) \sim\left(\sigma_{2} \rightarrow \sigma_{2}^{\prime}\right) \in \star / \mathrm{T}$ and $\Gamma \vdash\left(\lambda x: \sigma_{1} . e_{1}\right): \sigma_{1} \rightarrow \sigma_{1}^{\prime}$ and $\Gamma \vdash e_{2}: \sigma_{2}$. We have $\Gamma \vdash \gamma_{0}: \sigma_{2} \sim \sigma_{1} \in \star / \mathrm{T}$ and $\Gamma \vdash \gamma_{1}: \sigma_{1}^{\prime} \sim \sigma_{2}^{\prime} \in \star / \mathrm{T}$. Therefore, $\Gamma \vdash\left(\lambda x: \sigma_{1} .\left(e_{1} \triangleright \gamma_{1}\right)\right): \sigma_{1} \rightarrow \sigma_{2}^{\prime}$ and $\Gamma \vdash e_{2} \triangleright \gamma_{0}: \sigma_{1}$ and the result follows by the application typing rule.

- Case SSTPUSH: Straightforward use of inversion and typing/coercion rules.

- Case SSCPush: By inversion, $\Gamma \vdash \gamma:\left(\varphi_{1} \sim \varphi_{2}\right) \Rightarrow \sigma \sim\left(\varphi_{1}^{\prime} \sim \varphi_{2}^{\prime}\right) \Rightarrow \sigma^{\prime} \in \star / \mathrm{T}$ and $\Gamma \vdash \gamma^{\prime}: \varphi_{1}^{\prime} \sim \varphi_{2}^{\prime} \in \eta / \mathrm{C}$ and $\Gamma, c: \varphi_{1} \sim \varphi_{2} / \mathrm{C} \vdash e: \sigma$. We have $\Gamma \vdash \gamma_{0}: \varphi_{1} \sim \varphi_{1}^{\prime} \in \eta / \mathrm{C}$ and $\Gamma \vdash \gamma_{1}: \varphi_{2}^{\prime} \sim \varphi_{2} \in \eta / \mathrm{C}$ and $\Gamma \vdash \gamma_{2}: \sigma \sim \sigma^{\prime} \in \star / \mathrm{T}$. Therefore $\Gamma \vdash e \triangleright \gamma_{2}: \sigma^{\prime}$ and $\Gamma \vdash \gamma_{0} ; \gamma^{\prime} ; \gamma_{1}: \varphi_{1} \sim \varphi_{2} \in \eta / C$. Finally, the RHS has type $\sigma^{\prime}$ by coercion abstraction and application typing rules.

- Case SSKPUsh: By inversion, $\Gamma \vdash \gamma: T \bar{\varphi} \sim T \bar{\varphi}^{\prime} \in \star / T$ and $K: \forall \Delta . \sigma^{\prime} \in \Sigma$ and $\Gamma \vdash K \bar{\varphi} \triangleright \nabla \gamma: T \bar{\varphi}^{\prime}$. Further inversion yields $\Gamma \vdash K \bar{\varphi} \bar{\rho}: T \bar{\varphi}$ and $\Gamma \vdash \bar{\rho}: \Theta[\bar{a} \mapsto \bar{\varphi}]$ where $\sigma^{\prime}=\forall \Theta . T \bar{a}$. Let $\gamma=(\forall \Theta . T \bar{a})[\bar{a} \mapsto$ nth $\gamma]$ By lifting lemma, $\Gamma \vdash \gamma: \forall \Theta[\bar{a} \mapsto \bar{\varphi}] . T \bar{\varphi} \sim \forall \Theta\left[\bar{a} \mapsto \bar{\varphi}^{\prime}\right] . T \bar{\varphi}^{\prime} \in \star /$ T. Therefore we have by Lemma 11 that $\Gamma \vdash \bar{\rho} \triangleright \gamma: \Theta\left[\bar{a} \mapsto \bar{\varphi}^{\prime}\right]$ and thus $\Gamma \vdash K \bar{\varphi}^{\prime}(\bar{\rho} \triangleright \gamma): T \bar{\varphi}^{\prime}$

- Case TRAns: Application of CTRAns.

- All other cases by induction. 
Proof of THEOREM 15 (Progress): If $\Sigma$ is consistent and $\Sigma \vdash e_{1}: \sigma$ and $e_{1}$ is not a value $v$ or a coerced value $v \triangleright \gamma$, then there exists an $e_{2}$ such that $e_{1} \rightsquigarrow e_{2}$.

Proof by induction on $e_{1}$. Assume $e_{1}$ is not a value or a coerced value.

- Case $e_{1}=e e^{\prime}$. By induction, either $e$ is a value $v$ or a coerced value $v \triangleright \gamma$ or takes a step. In the first case, by canonical forms, $v$ is either an abstraction (which beta reduces) or a constructor application (which means that $e_{1}$ is a value). In the second case, we have a coercion $\gamma$ between a value type $\tau$ (the type of $v$ ) and $\sigma_{1} \rightarrow \sigma_{2}$. By consistency, then $\tau$ must be $\sigma_{1}^{\prime} \rightarrow \sigma_{2}^{\prime}$ and the push rule applies. In the last case $e_{1}$ steps by the application congruence rule.

- Case $e_{1}=e \varphi$ and $e_{1}=e \gamma$ are analogous to the previous case.

- Case $e_{1}=e \triangleright \gamma$. By induction, either $e$ is value $v$ or a coerced value $v \triangleright \gamma^{\prime}$ or takes a step. In the first case, then $e_{1}$ is a coerced value. In the second case, $\left(v \triangleright \gamma^{\prime}\right) \triangleright \gamma$ steps to $v \triangleright\left(\gamma^{\prime} ; \gamma\right)$. In the last case, the congruence rule for coercion apply.

- Case $e_{1}=$ case $_{\sigma} e$ of brs. By induction, either $e$ is a value $v$ or a coerced value $v \triangleright \gamma$ or takes a step. In the first case, by canonical forms, $v$ is a constructor application, so the case expression reduces. In the second case, we have a coercion $\gamma$ between a value type $\tau$ (the type of $v$ ) and $T \bar{\varphi}$. By consistency, then $\tau$ must be $T \bar{\varphi}^{\prime}$ and the push rule applies. In the last case $e_{1}$ steps by the case congruence rule.

\section{B.2 Rewriting}

LEMMA 32 (Rewriting regularity). If $\Gamma \vdash \varphi_{1} \rightsquigarrow \varphi_{2} \in \kappa$ then $\vdash \Gamma$ and $\Gamma \vdash \varphi_{1}: \kappa$ and $\Gamma \vdash \varphi_{2}: \kappa$.

Once we get a type constants at the head, it remains.

LEMma 33 (Constant Rewriting). If Good $\Gamma$ and $\Gamma \vdash T \bar{\varphi} \rightsquigarrow \varphi^{\prime} \in \eta / R$ then $\varphi^{\prime}=T \bar{\varphi}^{\prime}$ and $\Gamma \vdash \bar{\varphi} \rightsquigarrow \bar{\varphi}^{\prime} \in \bar{\kappa} / R$.

(Proof is by inspection of the rules of type rewriting.) There is a similar result for types of the form $\forall a: \kappa . \sigma$.

We can add types to the end of a list of arguments that reduce.

Lemma 34 (Snoc). If $\Gamma \vdash \bar{\varphi} \rightsquigarrow \bar{\varphi}^{\prime} \in \bar{\kappa} / R_{1}$ and $\Gamma \vdash \psi \rightsquigarrow \psi^{\prime} \in \eta / \min \left(R_{1}, R_{2}\right)$, then $\Gamma \vdash \bar{\varphi}, \psi \rightsquigarrow \bar{\varphi}^{\prime}, \psi^{\prime} \in\left(\bar{\kappa}, \eta / R_{2}\right) / R_{1}$

LEMma 35 (Single Rewriting Application). If Good $\Gamma$ and $\Gamma \vdash \varphi_{1} \rightsquigarrow \varphi_{1}^{\prime} \in\left(\eta_{1} / R_{1} \rightarrow \eta_{2}\right) / R_{2}$ and $\Gamma \vdash \varphi_{2} \rightsquigarrow \varphi_{2}^{\prime} \in \eta_{1} / \min \left(R_{1}, R_{2}\right)$ then $\Gamma \vdash \varphi_{1} \varphi_{2} \rightsquigarrow \varphi_{1}^{\prime} \varphi_{2}^{\prime} \in \eta_{2} / R_{2}$.

Proof Straightforward case analysis on the rewriting of $\varphi_{1}$.

LEMma 36 (Matching). If Good $\Gamma$ and $\Gamma \vdash c \bar{\psi}: H \bar{\varphi} \sim \sigma \in \eta / R$ and $\Gamma \vdash \bar{\varphi} \rightsquigarrow \bar{\varphi}^{\prime} \in \bar{\kappa}_{1} / R$ then there exist $\bar{\psi}^{\prime}$ and $\sigma^{\prime}$, such that $\Gamma \vdash c \bar{\psi}^{\prime}: H \bar{\varphi}^{\prime} \sim \sigma^{\prime} \in \eta / R$ and $\Gamma \vdash \sigma \rightsquigarrow \sigma^{\prime} \in \eta / R$.

Proof Follows by the definition of Good $\Gamma$ (first condition).

Proof OF Theorem 21: If Good $\Gamma$ and $\Gamma \vdash \varphi \rightsquigarrow \varphi_{1} \in \kappa$ and $\Gamma \vdash \varphi \rightsquigarrow \varphi_{2} \in \kappa$ then there exists a $\varphi_{3}$ such that $\Gamma \vdash \varphi_{1} \rightsquigarrow \varphi_{3} \in \kappa$ and $\Gamma \vdash \varphi_{2} \rightsquigarrow \varphi_{3} \in \kappa$.

Proof We prove this by induction on the size of $\varphi$. We proceed by inversion on the rules used to deduce that $\Gamma \vdash \varphi \rightsquigarrow \varphi_{1} \in \kappa$ and $\Gamma \vdash \varphi \rightsquigarrow \varphi_{2} \in \kappa$. Most of the cases are straightforward, and follow by the induction hypothesis, except for two combinations:

- Case RCONST-RRED. In this case we have that $\Gamma \vdash H \bar{\varphi} \rightsquigarrow H \bar{\varphi}^{\prime} \in \eta / R$ given that

$$
\Gamma \vdash \bar{\varphi} \rightsquigarrow \bar{\varphi}^{\prime} \in \bar{\kappa} / R
$$

Moreover, we let $\bar{\varphi}=\bar{\varphi}_{1}, \bar{\varphi}_{2}$ such that $\Gamma \vdash H \bar{\varphi} \rightsquigarrow \sigma \bar{\varphi}_{2}^{\prime \prime} \in \eta / R$ given that

$$
\begin{array}{r}
\Gamma \vdash \bar{\varphi}_{1} \rightsquigarrow \bar{\varphi}_{1}^{\prime \prime} \in \bar{\kappa}_{1} / R \\
\Gamma \vdash \bar{\varphi}_{2} \rightsquigarrow \bar{\varphi}_{2}^{\prime \prime} \in \bar{\kappa}_{2} / R \\
\Gamma \vdash c \bar{\psi}: H \bar{\varphi}_{1}^{\prime \prime} \sim \sigma \in\left(\bar{\kappa}_{2} \rightarrow \eta\right) / R
\end{array}
$$

By splitting $\bar{\varphi}^{\prime}$ accordingly, let us name the split $\bar{\varphi}_{1}^{\prime}, \bar{\varphi}_{2}^{\prime}$, it must then be:

$$
\begin{aligned}
& \Gamma \vdash \bar{\varphi}_{1} \rightsquigarrow \bar{\varphi}_{1}^{\prime} \in \bar{\kappa}_{1} / R \\
& \Gamma \vdash \bar{\varphi}_{2} \rightsquigarrow \bar{\varphi}_{2}^{\prime} \in \bar{\kappa}_{2} / R
\end{aligned}
$$

By using (2) with (5), and (3) with (5), and applying the induction hypothesis to each element in the corresponding vectors we get

$$
\begin{aligned}
& \Gamma \vdash \bar{\varphi}_{1}^{\prime} \rightsquigarrow \bar{\varphi}_{11} \in \bar{\kappa}_{1} / R \\
& \Gamma \vdash \bar{\varphi}_{1}^{\prime \prime} \rightsquigarrow \bar{\varphi}_{11} \in \bar{\kappa}_{1} / R \\
& \Gamma \vdash \bar{\varphi}_{2}^{\prime} \rightsquigarrow \bar{\varphi}_{22} \in \bar{\kappa}_{2} / R \\
& \Gamma \vdash \bar{\varphi}_{2}^{\prime \prime} \rightsquigarrow \bar{\varphi}_{22} \in \bar{\kappa}_{2} / R
\end{aligned}
$$

for some vectors $\bar{\varphi}_{11}$ and $\bar{\varphi}_{22}$.

We will be done if we can find some $\sigma^{\prime}$ for which we can show the following two statements:

$$
\begin{aligned}
\Gamma \vdash \sigma \bar{\varphi}_{2}^{\prime \prime} & \rightsquigarrow \sigma^{\prime} \bar{\varphi}_{22} \in \eta / R \\
\Gamma \vdash H \bar{\varphi}_{1}^{\prime} \bar{\varphi}_{2}^{\prime} & \rightsquigarrow \sigma^{\prime} \bar{\varphi}_{22} \in \eta / R
\end{aligned}
$$


For this, we will appeal to the matching lemma, and equations (4), (8). This lemma gives $\bar{\psi}^{\prime}$ and and $\sigma^{\prime}$ such that:

$$
\begin{array}{r}
\Gamma \vdash \sigma \rightsquigarrow \sigma^{\prime} \in\left(\bar{\kappa}_{2} \rightarrow \eta\right) / R \\
\Gamma \vdash c \bar{\psi}^{\prime}: H \bar{\varphi}_{11} \sim \sigma^{\prime} \in\left(\bar{\kappa}_{2} \rightarrow \eta\right) / R
\end{array}
$$

By appealing to (a straightforward extension of) the single application lemma, and using (13) and (9) we get our first goal, (11). For the second goal (12) we use (7), (9), (14), and apply rule RRED. Hence, this case is done.

- Case RRED-RRED. In this case we let $\bar{\varphi}=\bar{\varphi}_{1}, \bar{\varphi}_{2}$ such that $\Gamma \vdash H \bar{\varphi} \rightsquigarrow \sigma \bar{\varphi}_{2}^{\prime} \in \eta / R$ given that

$$
\begin{array}{r}
\Gamma \vdash \bar{\varphi}_{1} \rightsquigarrow \bar{\varphi}_{1}^{\prime} \in \bar{\kappa}_{1} / R \\
\Gamma \vdash \bar{\varphi}_{2} \rightsquigarrow \bar{\varphi}_{2}^{\prime} \in \bar{\kappa}_{2} / R \\
\Gamma \vdash c \bar{\psi}: H \bar{\varphi}_{1}^{\prime} \sim \sigma \in\left(\bar{\kappa}_{2} \rightarrow \eta\right) / R
\end{array}
$$

We let another possible split $\bar{\varphi}=\bar{\varphi}_{3}, \bar{\varphi}_{4}$ such that $\Gamma \vdash H \bar{\varphi} \rightsquigarrow \sigma_{0} \bar{\varphi}_{4}^{\prime} \in \eta / R$ given that

$$
\begin{array}{r}
\Gamma \vdash \bar{\varphi}_{3} \rightsquigarrow \bar{\varphi}_{3}^{\prime} \in \bar{\kappa}_{3} / R \\
\Gamma \vdash \bar{\varphi}_{4} \rightsquigarrow \bar{\varphi}_{4}^{\prime} \in \bar{\kappa}_{4} / R \\
\Gamma \vdash c^{\prime} \bar{\psi}^{\prime}: H \bar{\varphi}_{3}^{\prime} \sim \sigma_{0} \in\left(\bar{\kappa}_{4} \rightarrow \eta\right) / R
\end{array}
$$

By the induction hypothesis for every type in $\bar{\varphi}$, and (15), (16), (18), (19) we get that there exists a $\bar{\varphi}^{\prime \prime}$ such that

$$
\begin{aligned}
& \Gamma \vdash\left(\bar{\varphi}_{3}^{\prime}, \bar{\varphi}_{4}^{\prime}\right) \rightsquigarrow \bar{\varphi}^{\prime \prime} \in \bar{\kappa} / R \\
& \Gamma \vdash\left(\bar{\varphi}_{1}^{\prime}, \bar{\varphi}_{2}^{\prime}\right) \rightsquigarrow \bar{\varphi}^{\prime \prime} \in \bar{\kappa} / R
\end{aligned}
$$

where $\bar{\kappa}=\bar{\kappa}_{1}, \bar{\kappa}_{2}=\bar{\kappa}_{3}, \bar{\kappa}_{4}$. Now, consider the $1-2$ split and $3-4$ split induced on $\bar{\varphi}^{\prime \prime}=\bar{\varphi}_{1}^{\prime \prime}, \bar{\varphi}_{2}^{\prime \prime}=\bar{\varphi}_{3}^{\prime \prime}, \bar{\varphi}_{4}^{\prime \prime}$. By the matching lemma, and (20) we know that there exist $\bar{\psi}^{\prime \prime}$ such that $\Gamma \vdash c \bar{\psi}^{\prime \prime}: H \bar{\varphi}_{1}^{\prime \prime} \sim \sigma^{\prime \prime} \in\left(\bar{\kappa}_{2} \rightarrow \eta\right) / R$ and moreover $\Gamma \vdash \sigma \rightsquigarrow \sigma^{\prime \prime} \in\left(\bar{\kappa}_{2} \rightarrow \eta\right) / R$. Similarly, by the matching lemma and (21) we know that there exist $\bar{\psi}^{\prime \prime \prime}$ such that $\Gamma \vdash c^{\prime} \bar{\psi}^{\prime \prime \prime}: H \bar{\varphi}_{3}^{\prime \prime} \sim \sigma_{0}^{\prime \prime} \in\left(\bar{\kappa}_{4} \rightarrow \eta\right) / R$ with $\Gamma \vdash \sigma_{0} \rightsquigarrow \sigma_{0}^{\prime \prime} \in\left(\bar{\kappa}_{4} \rightarrow \eta\right) / R$. However, $\bar{\varphi}_{3}^{\prime \prime}$ and $\bar{\varphi}_{1}^{\prime \prime}$ come from the same vector $\bar{\varphi}^{\prime \prime}$ and hence, by appealing to the definition of good contexts, it must be the case that the $1-2,3-4$ split is exactly the same. Hence $\bar{\varphi}_{3}^{\prime \prime}=\bar{\varphi}_{1}^{\prime \prime}$ and similarly $\bar{\varphi}_{2}^{\prime \prime}=\bar{\varphi}_{4}^{\prime \prime}, c=c^{\prime}$, $\sigma^{\prime \prime}=\sigma_{0}^{\prime \prime}=\sigma^{\prime \prime \prime}$. I.e. we have:

$$
\begin{aligned}
\Gamma \vdash \sigma \rightsquigarrow \sigma^{\prime \prime \prime} & \in\left(\bar{\kappa}_{2} \rightarrow \eta\right) / R \\
\Gamma \vdash \sigma_{0} \rightsquigarrow \sigma^{\prime \prime \prime} & \in\left(\bar{\kappa}_{4} \rightarrow \eta\right) / R
\end{aligned}
$$

Hence, it suffices to show the following two:

$$
\begin{gathered}
\Gamma \vdash \sigma \bar{\varphi}_{2}^{\prime} \rightsquigarrow \sigma^{\prime \prime \prime} \bar{\varphi}_{2}^{\prime \prime} \in\left(\bar{\kappa}_{2} \rightarrow \eta\right) / R \\
\Gamma \vdash \sigma_{0} \bar{\varphi}_{4}^{\prime} \rightsquigarrow \sigma^{\prime \prime \prime} \bar{\varphi}_{4}^{\prime \prime} \in\left(\bar{\kappa}_{4} \rightarrow \eta\right) / R
\end{gathered}
$$

Since the $1-2$ split and the $3-4$ split are the same, the result will follow by the induction hypothesis for (15) with (18), and (16) with (19), reduction (23) and (24), and the single application lemma.

Proof of Lemma 20: If $\operatorname{Good} \Gamma$ and $\Gamma \vdash \varphi_{1} \Leftrightarrow \varphi_{1}^{\prime} \in\left(\eta_{1} / R_{1} \rightarrow \eta_{2}\right) / R_{2}$ and $\Gamma \vdash \varphi_{2} \Leftrightarrow \varphi_{2}^{\prime} \in \eta_{1} / \min \left(R_{1}, R_{2}\right)$ then $\Gamma \vdash \varphi_{1} \varphi_{2} \Leftrightarrow \varphi_{1}^{\prime} \varphi_{2}^{\prime} \in \eta_{2} / R_{2}$.

Proof Assume the intermediate join points of $\varphi_{1}$ and $\varphi_{1}^{\prime}$ and $\varphi_{2}$ and $\varphi_{2}^{\prime}$, call them $\sigma_{1}$ and $\sigma_{2}$ respectively. By applying multiple times the single application lemma and using reflexivity of rewriting we see that $\sigma_{1} \sigma_{2}$ must be a join point for $\varphi_{1} \varphi_{2}$ and $\varphi_{1}^{\prime} \varphi_{2}^{\prime}$.

Corollary 37 (Multi-Application). If Good $\Gamma$ and $\Gamma \vdash \varphi_{1} \Leftrightarrow \varphi_{1}^{\prime} \in(\bar{\kappa} \rightarrow \eta) / R$ and $\Gamma \vdash \bar{\varphi}_{2} \Leftrightarrow \bar{\varphi}_{2}^{\prime} \in \bar{\kappa} / R$ then $\Gamma \vdash \varphi_{1} \bar{\varphi}_{2} \Leftrightarrow$ $\varphi_{1}^{\prime} \bar{\varphi}_{2}^{\prime} \in \eta / R$.

Lemma 38 (StepSubst1). If Good $\Gamma$ and $\Gamma, a: \kappa, \Delta \vdash \sigma: \eta / R$ and $\Gamma \vdash \varphi \rightsquigarrow \varphi^{\prime} \in \kappa$, then $\Gamma \vdash \sigma[a \mapsto \varphi] \Leftrightarrow \sigma\left[a \mapsto \varphi^{\prime}\right] \in \eta / R$.

Proof by induction on $\sigma$.

Case $\sigma=F \bar{\varphi}$ : By induction, we have $\Gamma, \Delta \vdash \bar{\varphi}[a \mapsto \varphi] \Leftrightarrow \bar{\varphi}\left[a \mapsto \varphi^{\prime}\right] \in \bar{\kappa}_{1} / R$. Result holds by the appliation lemma.

Case $\sigma=b \bar{\varphi}$ : By induction, we have $\Gamma, \Delta \vdash \bar{\varphi}[a \mapsto \varphi] \Leftrightarrow \bar{\varphi}\left[a \mapsto \varphi^{\prime}\right] \in \bar{\kappa}_{1} / R$. If $b$ is not $a$, we are done by the application lemma. Otherwise, suppose $a=b$ : so we want to show that $\Gamma, \Delta \vdash \varphi(\bar{\varphi}[a \mapsto \varphi]) \Leftrightarrow \varphi\left(\bar{\varphi}\left[a \mapsto \varphi^{\prime}\right]\right) \in \kappa$. This also holds by the application lemma.

Case $\sigma=T \bar{\varphi}$ : By induction and the application lemma.

Case $\sigma=\forall b: \kappa . \sigma^{\prime}$ : Result holds by induction.

LEMma 39 (StepSubstMany). If Good $\Gamma$ and $\Gamma, a: \kappa, \Delta \vdash \sigma: \kappa^{\prime}$ and $\Gamma \vdash \varphi \rightsquigarrow^{*} \varphi^{\prime} \in \kappa$, then $\Gamma \vdash \sigma[a \mapsto \varphi] \Leftrightarrow \sigma\left[a \mapsto \varphi^{\prime}\right] \in \kappa^{\prime}$.

Proof Proof is by induction on the number of steps in $\Gamma \vdash \varphi \rightsquigarrow^{*} \varphi^{\prime} \in \kappa$. If $n=0$ then the result is trivial. Say $n=m+1$ and $\Gamma \vdash \varphi \rightsquigarrow \varphi^{\prime \prime} \in \kappa$ and $\Gamma \vdash \varphi^{\prime \prime} \rightsquigarrow^{*} \varphi^{\prime} \in \kappa$ in $m$ steps. By induction, we have $\Gamma \vdash \sigma\left[a \mapsto \varphi^{\prime \prime}\right] \Leftrightarrow \sigma\left[a \mapsto \varphi^{\prime}\right] \in \kappa^{\prime}$. We just need to show that $\Gamma \vdash \sigma[a \mapsto \varphi] \Leftrightarrow \sigma\left[a \mapsto \varphi^{\prime \prime}\right] \in \kappa^{\prime}$. However, this result holds by lemma StepSubst1.

LEMma 40 (StepSubst2). If Good $\Gamma$ and $\Gamma, a: \kappa, \Delta \vdash \sigma \rightsquigarrow \sigma^{\prime} \in \kappa^{\prime}$ and $\Gamma \vdash \varphi: \kappa$, then $\Gamma, \Delta \vdash \sigma[a \mapsto \varphi] \Leftrightarrow \sigma\left[a \mapsto \varphi^{\prime}\right] \in \kappa^{\prime}$.

Proof is by induction on $\sigma$, appealing to Application. 
LEMmA 41 (Transitivity of rewriting). If $\operatorname{Good} \Gamma$ and $\Gamma \vdash \varphi_{1} \Leftrightarrow \varphi_{2} \in \kappa$ and $\Gamma \vdash \varphi_{2} \Leftrightarrow \varphi_{3} \in \kappa$ then $\Gamma \vdash \varphi_{1} \Leftrightarrow \varphi_{3} \in \kappa$.

Proof Assume that $\Gamma \vdash \varphi_{1} \rightsquigarrow^{*} \sigma \in \kappa$ and $\Gamma \vdash \varphi_{2} \rightsquigarrow^{*} \sigma \in \kappa$ in $n_{1}$ and $n_{2}$ steps respectively. Similarly, $\Gamma \vdash \varphi_{2} \rightsquigarrow{ }^{*} \sigma^{\prime} \in \kappa$ and $\Gamma \vdash \varphi_{3} \rightsquigarrow^{*} \sigma^{\prime} \in \kappa$ in $n_{2}$ and $n_{3}$ steps respectively. Because of reflexivity of rewritting we can extend all those reductions so that each takes exactly $m=\max \left(n_{1}, n_{2}, n_{3}, n_{4}\right)$ steps. The result then follows by the local diamond property.

Proof of Lemma 22 If Good $\Gamma$ and $\Gamma, a: \kappa, \Delta \vdash \sigma \rightsquigarrow^{*} \sigma^{\prime} \in \kappa^{\prime}$ and $\Gamma \vdash \varphi \rightsquigarrow^{*} \varphi^{\prime} \in \kappa$, then there is some $\Gamma, \Delta \vdash \sigma[a \mapsto \varphi] \Leftrightarrow$ $\sigma^{\prime}\left[a \mapsto \varphi^{\prime}\right] \in \kappa^{\prime}$ : :

Proof is by induction on the number of steps in $\Gamma, a: \kappa, \Delta \vdash \sigma \rightsquigarrow^{*} \sigma^{\prime} \in \kappa^{\prime}$. If $n=0$, then the result follows from StepSubstMany. If $n=m+1$, suppose $\Gamma, a: \kappa, \Delta \vdash \sigma \rightsquigarrow \sigma^{\prime \prime} \in \kappa^{\prime}$ and $\Gamma, a: \kappa, \Delta \vdash \sigma^{\prime \prime} \rightsquigarrow{ }^{*} \sigma^{\prime} \in \kappa^{\prime}$ in $m$ steps. By induction, we have $\Gamma, \Delta \vdash \sigma^{\prime \prime}[a \mapsto \varphi] \Leftrightarrow \sigma^{\prime}\left[a \mapsto \varphi^{\prime}\right] \in \kappa^{\prime}$. We just need to show that $\Gamma \vdash \sigma[a \mapsto \varphi] \Leftrightarrow \sigma^{\prime \prime}[a \mapsto \varphi] \in \kappa^{\prime}$. However, this result holds by lemma StepSubst2.

Proof OF LemMA 23 If Good $\Gamma$ and $\Gamma \vdash \gamma: \varphi_{1} \sim \varphi_{2} \in \kappa$ then $\Gamma \vdash \varphi_{1} \Leftrightarrow \varphi_{2} \in \kappa$. :

Proof is by induction on $\gamma$.

- $\gamma=\varphi$. Trivial as $\varphi_{1}=\varphi_{2}$.

- $\gamma=\gamma_{1} \gamma_{2}$. Holds by induction and application lemma above.

- $\gamma=\forall a: \kappa \cdot \gamma^{\prime}$. Holds by induction and RALL.

- $\gamma=c \bar{\psi}$ where $\kappa=\eta / R$. We have $\varphi_{1}=\sigma_{1}[\bar{a} \mapsto \bar{\psi}]$ and $\varphi_{2}=\sigma_{2}[\bar{a} \mapsto \bar{\psi}]$. We also must have $\Gamma, \Delta \vdash \sigma_{1} \rightsquigarrow \sigma_{2} \in \eta / R$ (by noting that type variables are normal and instantiating $c$ with variables in $\bar{\kappa}$.) The desired result holds by the substitution lemmas and transitivity.

- $\gamma=\mathbf{s y m} \gamma^{\prime}$ Trivial.

- $\gamma=\gamma_{1} ; \gamma_{2}$ Holds by transitivity of rewriting.

- $\gamma=\mathbf{n t h} k \gamma^{\prime}$ By inversion we have $\Gamma \vdash \gamma^{\prime}: T \bar{\varphi}_{1} \sim T \bar{\varphi}_{2} \in \eta / \mathrm{T}$. We want to show that there is some $\varphi$ such that, $\Gamma \vdash n t h k \bar{\varphi}_{1} \rightsquigarrow * \varphi \in n t h k \bar{\kappa}$ and $\Gamma \vdash n t h k \bar{\varphi}_{2} \rightsquigarrow * \varphi \in n t h k \bar{\kappa}$.

By induction we have $\varphi$, such that $\Gamma \vdash T \bar{\varphi}_{1} \rightsquigarrow^{*} \varphi \in \eta / T$ and $\Gamma \vdash T \bar{\varphi}_{2} \rightsquigarrow^{*} \varphi \in \eta / \mathrm{T}$. By Constant Rewriting, we have $\varphi=T \bar{\varphi}^{\prime}$, and $\Gamma \vdash \bar{\varphi}_{1} \rightsquigarrow^{*} \bar{\varphi}^{\prime} \in \bar{\kappa} / \mathrm{T}$ and $\Gamma \vdash \bar{\varphi}_{2} \rightsquigarrow^{*} \bar{\varphi}^{\prime} \in \bar{\kappa} / \mathrm{T}$. By inversion of these two results, we get the appriate $\varphi$.

- $\gamma=\gamma_{1} @ \psi$ By inversion we have $\Gamma \vdash \gamma_{1}: \forall a: \kappa . \sigma_{1} \sim \forall a: \kappa . \sigma_{2} \in \star / \mathrm{T}$ and $\Gamma \vdash \psi: \kappa$. We want to show that there is some $\varphi$, such that $\Gamma \vdash \sigma_{1}[a \mapsto \psi] \rightsquigarrow^{*} \varphi \in \star / T$ and $\Gamma \vdash \sigma_{2}[a \mapsto \psi] \rightsquigarrow^{*} \varphi \in \star / \mathrm{T}$. By induction, we know that there is some $\sigma$ and $\varphi^{\prime}$, such that $\Gamma \vdash \forall a: \kappa . \sigma_{1} \rightsquigarrow * \forall a: \kappa . \sigma \in \star / \mathrm{T}$ and $\Gamma \vdash \forall a: \kappa . \sigma_{2} \rightsquigarrow{ }^{*} \forall a: \kappa . \sigma \in \star / \mathrm{T}$. The substitution lemma and transitivity gives us the desired result. 